\title{
A Computational Model of Levodopa-Induced Toxicity in Substantia Nigra Pars Compacta in Parkinson's Disease
}

Vignayanandam Ravindernath Muddapu ${ }^{1}$, Karthik Vijayakumar ${ }^{2}$, Keerthiga Ramakrishnan $^{3}$, V Srinivasa Chakravarthy ${ }^{1, \#}$

${ }^{1}$ Department of Biotechnology, Bhupat and Jyothi Mehta School of Biosciences, Indian Institute of Technology Madras, Chennai 600036, Tamil Nadu, India.

${ }^{2}$ Department of Biotechnology, Rajalakshmi Engineering College, Chennai 602105, Tamil Nadu, India.

${ }^{3}$ Division of Psychiatry and Applied Psychology, School of Medicine, University of Nottingham, Nottingham NG8 1BB, United Kingdom.

\section{\# Corresponding Author:}

Prof V. Srinivasa Chakravarthy, Ph.D.

Room 505, Block 1, Department of Biotechnology,

Bhupat and Mehta Jyothi School of Biosciences,

Indian Institute of Technology Madras, Adyar,

Chennai 600036, Tamil Nadu, India.

Tel: +914422574115

Fax: +914422574102

Email: schakra@ee.iitm.ac.in

Running Title: Levodopa-Induced Toxicity in SNc 


\title{
A Computational Model of Levodopa-Induced Toxicity in Substantia Nigra Pars Compacta in Parkinson's Disease
}

\begin{abstract}
Background: Parkinson's disease (PD) is caused by the progressive loss of dopaminergic cells in substantia nigra pars compacta ( $\mathrm{SNc}$ ). The root cause of this cell loss in PD is still not decisively elucidated. A recent line of thinking traces the cause of PD neurodegeneration to metabolic deficiency. Due to exceptionally high energy demand, SNc neurons exhibit a higher basal metabolic rate and higher oxygen consumption rate, which results in oxidative stress. Recently, we have suggested that the excitotoxic loss of SNc cells might be due to energy deficiency occurring at different levels of neural hierarchy. Levodopa (LDOPA), a precursor of dopamine, which is used as a symptom-relieving treatment for PD, leads to outcomes that are both positive and negative. Several researchers suggested that LDOPA might be harmful to $\mathrm{SNc}$ cells due to oxidative stress. The role of LDOPA in the course of PD pathogenesis is still debatable.
\end{abstract}

New Method: We hypothesize that energy deficiency can lead to LDOPA-induced toxicity (LIT) in two ways: by promoting dopamine-induced oxidative stress and by exacerbating excitotoxicity in SNc. We present a multiscale computational model of SNc-striatum system, which will help us in understanding the mechanism behind neurodegeneration postulated above and provides insights for developing disease-modifying therapeutics.

Results: It was observed that SNc terminals are more vulnerable to energy deficiency than SNc somas. During LDOPA therapy, it was observed that higher LDOPA dosage results in increased loss of somas and terminals in SNc. It was also observed that co-administration of LDOPA and glutathione (antioxidant) evades LDOPA-induced toxicity in SNc neurons.

Comparison with Existing Methods: Our proposed multiscale model of SNc-striatum system is first of its kind, where SNc neuron was modelled at biophysical level, and striatal neurons were modelled at spiking level.

Conclusions: We show that our proposed model was able to capture LDOPA-induced toxicity in $\mathrm{SNc}$, caused by energy deficiency.

Keywords: Parkinson's disease; Levodopa; Dopamine; Substance P; Striatum; Substantia nigra pars compacta; 


\section{INTRODUCTION}

Almost all neurodegenerative diseases have a characteristic loss of a certain special type of cells that are vulnerable to death due to metabolic deficiency (Fu et al., 2018; Muddapu et al., 2020). Parkinson's disease (PD) is characterized by loss of dopaminergic neurons in the substantia nigra pars compacta $(\mathrm{SNc})$, which results in cardinal symptoms such as tremor, rigidity, bradykinesia and postural instability (Goldman and Postuma, 2014). The root cause of SNc cell loss in PD is still not decisively elucidated. Recently, a modeling study has been proposed where PD is described to be resulting from the metabolic deficiency in SNc (Muddapu et al., 2019; Muddapu and Chakravarthy, 2020a). The vulnerable cells of SNc are projection neurons with large axonal arbors of complex morphologies, requiring a huge amount of energy to maintain information processing activities (Bolam and Pissadaki, 2012; Giguère et al., 2019; Muddapu et al., 2020; Pissadaki and Bolam, 2013). Due to huge energy requirements, $\mathrm{SNc}$ neurons exhibit higher basal metabolic rates and higher oxygen consumption rate, which result in oxidative stress (Pacelli et al., 2015). With the help of a computational model, Muddapu et al. (Muddapu et al., 2019) have recently suggested that the excitotoxic loss of SNc cells might be due to energy deficiency occurring at different levels of neural hierarchy - systems, cellular and subcellular.

Levodopa (LDOPA), a precursor of dopamine (DA), is used as a symptom-relieving treatment for PD (Poewe et al., 2010). The usage of LDOPA for PD is still debated due to its side-effects with long-term treatment (Fahn, 2005; Lipski et al., 2011; Thanvi and Lo, 2004). Several researchers suggested that LDOPA might be harmful to SNc cells by a mechanism that probably involves oxidative stress (Carvey et al., 1997; Pardo et al., 1995; Takashima et al., 1999). However, several others proposed that LDOPA might not accentuate neurodegeneration of SNc neurons (Billings et al., 2019; Fahn, 2005; Fahn et al., 2004; Jenner and Brin, 1998) and sometimes acts a neuroprotective agent (Fahn, 2005; Schapira, 2008; Shimozawa et al., 2019) or promote recovery of dopaminergic markers in the striatum (Murer et al., 1998, 1999). After several studies, it is still not clear why LDOPA is not toxic in case of nonparkinsonian human subjects and healthy animals and toxic in PD models of rodents (Agid, 1998; Fahn, 1997; Lipski et al., 2011; Müller et al., 2004; Olanow and Obeso, 2011; Paoletti et al., 2019; Weiner, 2006; Ziv et al., 1997). The beneficial or toxic effects of LDOPA needs to be investigated with more thorough experiments performed at preclinical and clinical levels. 
In this paper, we investigate, using computational modelling, the hypothesis that LDOPA-induced toxicity can occur in two ways: by promoting DA-induced oxidative stress (autoxidation-relevant) (Borah and Mohanakumar, 2010; Carvey et al., 1997; Melamed et al., 1998; Pardo et al., 1995; Takashima et al., 1999; Walkinshaw and Waters, 1995) or by exacerbating excitotoxicity in SNc (autoxidation-irrelevant) (Blomeley et al., 2009; Blomeley and Bracci, 2008; Cheng et al., 1996; Pardo et al., 1993; Thornton and Vink, 2015), or by both the mechanisms which might be actually precipitated by energy deficiency. In order to investigate our hypothesis, we propose a multiscale computational model of L-DOPA-induced toxicity in SNc, which will help us in understanding the mechanism behind neurodegeneration due to LDOPA and gives insights for developing disease-modifying therapeutics.

\section{MATERIALS AND METHODS}

The proposed model of levodopa-induced toxicity (LIT) consists of the cortico-basal ganglia system. We model a part of the basal ganglia system comprising the following nuclei: SNc, Striatum, Subthalamic nucleus (STN), and Globus Pallidus externa (GPe). Within the SNc, we separately consider SNc soma (cell body) and SNc terminals (boutons) that make contact with striatal neurons. Within the striatum, we model D1-type receptor-expressing medium spiny neurons of two subtypes: 1) D1-MSNs that release GABA only (D1-MSN) (GABAergic only) and 2) D1-MSNs that release GABA and substance P, D1-MSN (GABAergic (G), and substance $\mathrm{P}(\mathrm{S}))$. In the cortex (CTX), the pyramidal neurons are modeled. Neurons in each nucleus are arranged as a two-dimensional lattice (Figure 1). All the simulations were performed by numerical integration using MATLAB (RRID: SCR_001622) with a time step of $0.1 \mathrm{~ms}$.

To implement energy deficiency conditions in the proposed model, the glucose and oxygen inputs were reduced to $\mathrm{SNc}$ cells. As the number of $\mathrm{SNc}$ neurons under energy deficiency increases, the dopaminergic tone to striatum decreases due to SNc terminal loss. The dopamine deficiency leads to lesser excitation of MSN neurons by pyramidal neurons in the cortex; as a result, SNc neurons get disinhibited. Disinhibition from MSN leads to overactivity of $\mathrm{SNc}$ neurons, which in turn results in $\mathrm{SNc}$ neurons degeneration due to excitotoxicity.

In order to examine the LDOPA role in the degeneration of SNc neurons in PD, we administer LDOPA after a certain percentage of SNc neuronal loss due to energy deficiency and investigate how LDOPA changes the course of SNc cell loss. 


\subsection{Izhikevich (Spiking) Neuronal Model (STN, GPe, MSN, CTX)}

The Izhikevich neuronal models are capable of exhibiting biologically realistic firing patterns at a relatively low computational expense (Izhikevich, 2003). The proposed model of LIT consists of MSN (D1-MSN (G) and D1-MSN (GS)), STN, GPe, and CTX are modeled as spiking neuronal models using Izhikevich neuronal models arranged in the form of twodimensional lattices (Figure 1). Based on the anatomical data of the rat basal ganglia (Supplementary material-1), the neuronal population sizes in the model were selected (Arbuthnott and Wickens, 2007; Oorschot, 1996). The Izhikevich parameters for MSN were adapted from (Humphries et al., 2009); for STN and GPe they were adapted from (Michmizos and Nikita, 2011) and (Mandali et al., 2015) respectively and those of CTX were adapted from (Izhikevich, 2003) are given in Supplementary material-2. The external bias current $\left(I^{x}\right)$ was adjusted to match the firing rate of nuclei with published data (Tripathy et al., 2015).

The Izhikevich neuronal model of STN, GPe, CTX, and MSN consists of two variables, membrane potential $\left(v^{x}\right)$ and membrane recovery variable $\left(u^{x}\right)$ :

$$
\begin{gathered}
C^{x} * \frac{d\left(v_{i j}^{x}\right)}{d t}=\varphi_{1}-u_{i j}^{x}+I_{i j}^{x}+I_{i j}^{s y n} \\
\frac{d\left(u_{i j}^{x}\right)}{d t}=a\left(b * \varphi_{2}-u_{i j}^{x}\right) \\
\text { if } x=S T N \text { or GPe or CTX, then } \\
\varphi_{1}=0.04\left(v_{i j}^{x}\right)^{2}+5 v_{i j}^{x}+140 \\
\varphi_{2}=v_{i j}^{x} \\
\text { if } x=M S N, \quad \text { then } \\
\varphi_{1}=k^{x}\left(v_{i j}^{x}-v_{r}^{x}\right)\left(v_{i j}^{x}-v_{t}^{x}\right) \\
\varphi_{2}=\left(v_{i j}^{x}-v_{r}^{x}\right)
\end{gathered}
$$

Resetting:

$$
\text { if } v_{i j}^{x} \geq v_{\text {peak }}^{x}, \quad \text { then } \quad\left\{\begin{array}{c}
v_{i j}^{x} \leftarrow c \\
u_{i j}^{x} \leftarrow u_{i j}^{x}+d
\end{array}\right\}
$$


where, $v_{i j}^{x}, u_{i j}^{x}, I_{i j}^{x}$, and $I_{i j}^{s y n}$ are the membrane potential, the membrane recovery variable, the external bias current, and total synaptic current received to neuron $x$ at the location $(i, j)$ respectively; $v_{t}^{x}$ and $v_{r}^{x}$ are the threshold and resting potentials respectively; $k^{x}$ is the membrane constant, $C^{x}$ is the membrane capacitance, $\{a, b, c, d\}$ are Izhikevich parameters; $v_{\text {peak }}^{x}$ is the maximum membrane voltage set to neuron with $x$ being GPe or CTX or STN or MSN neuron.

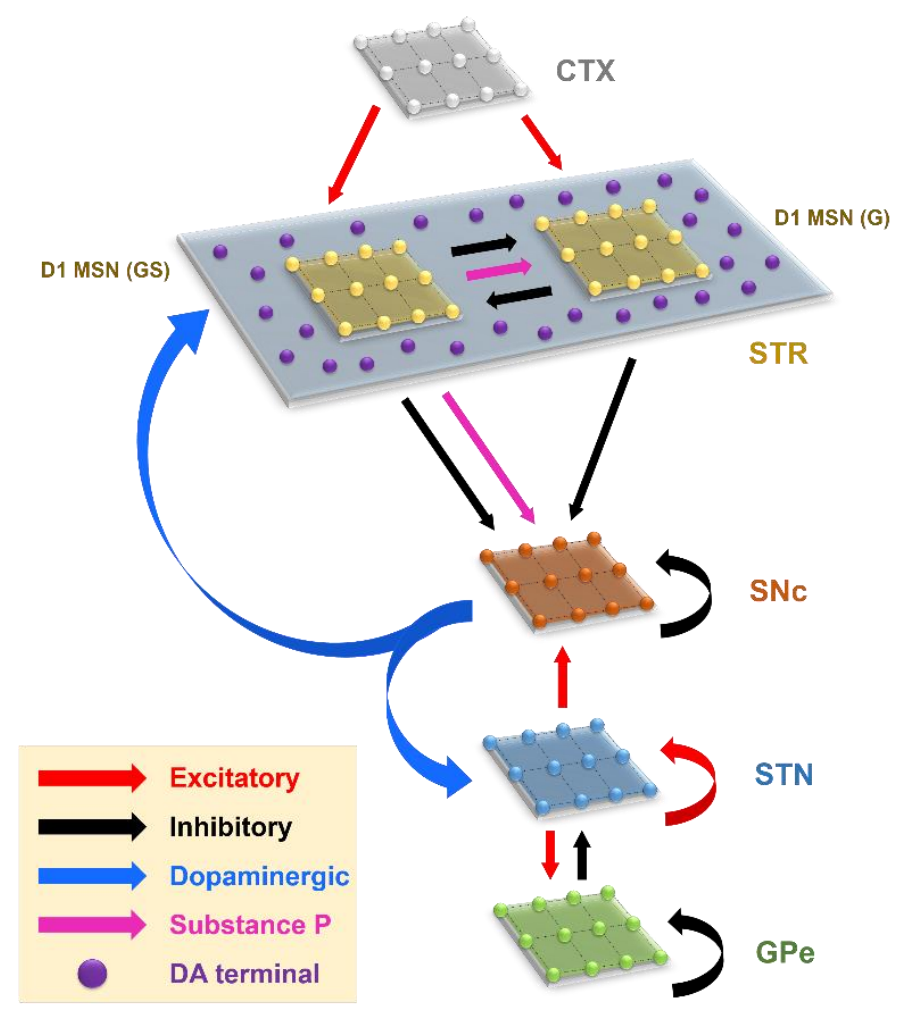

Figure 1: Model architecture of the levodopa-induced toxicity. CTX, cortex; STR, striatum; D1 MSN (GS), D1-type medium spiny neuron (GABAergic and Substance P); D1 MSN (G), D1-type medium spiny neuron (GABAergic); SNc, substantia nigra pars compacta; STN, subthalamic nucleus; GPe, globus pallidus externa; DA, dopamine.

\subsection{Biophysical (Conductance-based) Neuronal Model (SNc soma)}

The biophysical neuronal model of $\mathrm{SNc}$ in the proposed LIT model was adapted from (Muddapu and Chakravarthy, 2020b). The detailed biophysical model of SNc neuron consists of soma and terminal which includes cellular and molecular processes such as ion channels (including active ion pumps, ion exchangers), calcium buffering mechanism (calcium-binding proteins, calcium sequestration organelles (such as endoplasmic reticulum, mitochondria)), energy metabolism (glycolysis and oxidative phosphorylation), DA turnover processes 
(synthesis, storage, release, reuptake, and metabolism), molecular pathways involved in PD pathology (ROS formation and alpha-synuclein aggregation), and apoptotic pathways. The dynamics of SNc membrane potential $\left(v^{S N c}\right)$ is given as,

$$
\frac{d\left(v_{i j}^{S N c}\right)}{d t}=\frac{F * v_{c y t}}{C^{S N C} * \mathcal{A}_{p m u}} *\left[J_{m, N a}+2 * J_{m, C a}+J_{m, K}+J_{i n p}\right]
$$

where, $F$ is the Faraday's constant, $C^{S N c}$ is the $\mathrm{SNc}$ membrane capacitance, $v_{c y t}$ is the cytosolic volume, $\mathcal{A}_{p m u}$ is the cytosolic area, $J_{m, N a}$ is the sodium membrane ion flux, $J_{m, C a}$ is the calcium membrane ion flux, $J_{m, K}$ is the potassium membrane ion flux, $J_{\text {inp }}$ is the overall input current flux. The detailed information about the SNc neuron model was provided in (Muddapu and Chakravarthy, 2020b).

\subsection{Biochemical Dopamine Terminal Model (SNc terminal)}

The biochemical DA terminal of SNc in the proposed LIT model was adapted from (Muddapu and Chakravarthy, 2020b). The biochemical model of DA terminal consists of DA turnover processes, energy metabolism, and molecular pathways involved in PD pathology. The terminal is divided into two compartments, namely intracellular (cytoplasmic and vesicular) and extracellular compartments. The DA dynamics in the extracellular compartment $\left(\left[D A_{e}\right]\right)$ was modeled as,

$$
\frac{d\left(\left[D A_{e}\right]\right)}{d t}=J_{r e l}-J_{D A T}-J_{e d a}^{o}
$$

where, $J_{\text {rel }}$ represents the flux of calcium-dependent DA release from the DA terminal, $J_{D A T}$ represents the unidirectional flux of DA translocated from the extracellular compartment (ECS) into the intracellular compartment (cytosol) via DA plasma membrane transporter (DAT), and $J_{\text {eda }}^{o}$ represents the outward flux of DA degradation which clears DA from ECS.

The DA dynamics in the intracellular compartment $\left(\left[D A_{i}\right]\right)$ was modeled as

$$
\frac{d\left(\left[D A_{i}\right]\right)}{d t}=\frac{d\left(\left[D A_{c}\right]\right)}{d t}+\frac{d\left(\left[D A_{v}\right]\right)}{d t}
$$


where, $\left[D A_{c}\right]$ and $\left[D A_{v}\right]$ refer to the DA concentrations in the cytosolic and vesicular compartments, respectively.

The DA dynamics in the cytosolic compartment $\left(\left[D A_{c}\right]\right)$ is given by,

$$
\frac{d\left(\left[D A_{c}\right]\right)}{d t}=J_{D A T}-J_{V M A T}-J_{c d a}^{o}+J_{l d o p a}
$$

where, $J_{D A T}$ represents the unidirectional flux of DA translocated from ECS into the cytosol through DAT, $J_{V M A T}$ represents the flux of DA into vesicle through vesicular monoamine transporters (VMAT), $J_{i d a}^{o}$ represents the outward flux of DA degradation which clears DA from the cytosol, $J_{\text {ldopa }}$ represents the flux of synthesized cytosol DA from levodopa which is induced by calcium.

The DA dynamics in the vesicular compartment $\left(\left[D A_{v}\right]\right)$ is given by,

$$
\frac{d\left(\left[D A_{v}\right]\right)}{d t}=J_{V M A T}-J_{r e l}
$$

where, $J_{r e l}$ represents the flux of calcium-dependent DA release from the DA terminal, $J_{V M A T}$ represents the flux of DA stored into a vesicle.

Based on the membrane activity, the DA turnover and other molecular processes were modulated in the terminal. The modulation of neuronal activity on the terminal was carried on by calcium dynamics, where calcium modulates DA synthesis and release. The calciuminduced synthesis of DA is given as,

$$
J_{\text {ldopa }}=f\left(\left[C a_{i}\right]\right)
$$

The calcium-induced release of DA is given as,

$$
J_{\text {rel }}=f\left(\left[C a_{i}\right]\right)
$$

where, $\left[\mathrm{Ca}_{i}\right]$ is the intracellular calcium concentration in the DA terminal. For more details of the SNc terminal model, the reader may refer to (Muddapu and Chakravarthy, 2020b).

\subsection{Synaptic Connections}


The synaptic connectivity among different neuronal populations was modelled as a standard single exponential model of postsynaptic currents (Humphries et al., 2009) as follows:

$$
\begin{gathered}
\tau_{\text {Recep }} * \frac{d\left(h_{i j}^{x \rightarrow y}\right)}{d t}=-h_{i j}^{x \rightarrow y}+S_{i j}^{x}(t) \\
I_{i j}^{x \rightarrow y}(t)=W_{x \rightarrow y} * h_{i j}^{x \rightarrow y}(t) *\left(E_{\text {Recep }}-v_{i j}^{y}(t)\right)
\end{gathered}
$$

The N-Methyl-D-aspartic Acid (NMDA) current was regulated by voltage-dependent magnesium channels which were modelled as,

$$
B_{i j}\left(v_{i j}\right)=\frac{1}{1+\left(\frac{\left[M g^{2+}\right]}{3.57} * e^{-0.062 * v_{i j}^{y}(t)}\right)}
$$

where, $h_{i j}^{x \rightarrow y}$ is the gating variable for the synaptic current from $x$ to $y, \tau_{\text {Recep }}$ is the decay constant for the synaptic receptor, $S_{i j}^{x}$ is the spiking activity of neuron $x$ at time $t, W_{x \rightarrow y}$ is the synaptic weight from neuron $x$ to $y, v_{i j}^{y}$ is the membrane potential of the neuron $y$ for the neuron at the location $(i, j), E_{\text {Recep }}$ is the receptor-associated synaptic potential $($ Recep $=$ NMDA/AMPA/GABA), and $\left[\mathrm{Mg}^{2+}\right]$ is the magnesium ion concentration. The time constants of Gamma-Amino Butyric Acid (GABA), Alpha-amino-3-hydroxy-5-Methyl-4-isoxazole Propionic Acid (AMPA) and NMDA in GPe, CTX, MSN, SNc, and STN were chosen from (Götz et al., 1997) as given in Supplementary material-2.

To accommodate extensive axonal arborization of SNc neurons (Bolam and Pissadaki, 2012), we considered one-to-many projections from SNc soma to SNc terminals (Supplementary material-3). The connectivity patterns among different neuronal populations were given in Supplementary material-3.

\subsection{Lateral Connections}

The lateral connections in SNc, STN and GPe, were modelled as Gaussian neighborhoods (Muddapu et al., 2019),

$$
w_{i j, p q}^{m \rightarrow m}=A_{m} * e^{\frac{-d_{i j, p q}^{2}}{R_{m}^{2}}}
$$




$$
d_{i j, p q}^{2}=(i-p)^{2}+(j-q)^{2}
$$

where, $w_{i j, p q}^{m \rightarrow m}$ is the lateral connection weight of neuron type $m$ at the location $(i, j), d_{i j, p q}$ is the distance from center neuron $(p, q), R_{m}$ is the variance of Gaussian, $A_{m}$ is the strength of lateral synapse, $m=$ GPe or STN or SNC.

The connections within SNc and GPe populations were considered as inhibitory and within STN as excitatory (Muddapu et al., 2019) (Figure 1). No lateral connections were considered for both the MSNs and CTX populations. The lateral currents in the STN and GPe were modelled similar to equations (13-15) and in the case of SNc which was modelled as,

$$
\begin{gathered}
H_{\infty}=\frac{1}{1+e^{\left(\frac{-\left(v_{i j}^{x}-\theta_{g}-\theta_{g}^{H}\right)}{\sigma_{g}^{H}}\right)}} \\
\frac{d\left(s_{i j}^{x \rightarrow y}\right)}{d t}=\alpha *\left(1-s_{i j}^{x \rightarrow y}\right) * H_{\infty}-\beta * s_{i j}^{x \rightarrow y} \\
I_{i j}^{x \rightarrow y}(t)=W_{x \rightarrow y} * s_{i j}^{x \rightarrow y} *\left(v_{i j}^{y}(t)-E_{G A B A}\right)
\end{gathered}
$$

where, $I_{i j}^{x \rightarrow y}$ is the synaptic current from neuron $x$ to $y, W_{x \rightarrow y}$ is the synaptic conductance from neuron $x$ to $y, v_{i j}^{x}$ and $v_{i j}^{y}$ are the membrane potential of the neuron $x$ and $y$ respectively for the neuron at the location $(i, j), E_{G A B A}$ is the GABAergic receptor potential, $s_{i j}^{x \rightarrow y}$ is the synaptic gating variable for the neuron. The parametric values of $\alpha, \beta, \theta_{g}, \theta_{g}^{H}, \sigma_{g}^{H}$ were adapted from (Rubin and Terman, 2004) and given in Supplementary material-4.

\subsection{Neuromodulatory Effect on the Neuronal Populations}

The effect of neuromodulators such as DA and substance P (SP) in the proposed LIT model was modelled based on (Muddapu et al., 2019) and (Buxton et al., 2017), respectively.

\subsubsection{Dopaminergic modulation:}

DA-modulated lateral connection strength in SNc, STN, and GPe populations. As DA level increases, the lateral connection strength in SNc and GPe increases, whereas, in the case of STN, it decreases. DA-modulation of lateral connection strength was modelled as, 


$$
\begin{aligned}
& A^{S T N}=s_{\max }^{S T N} * e^{\left(-c d_{s t n} * D A_{S}(t)\right)} \\
& A^{G P e}=s_{\min }^{G P e} * e^{\left(c d_{\text {gpe }} * D A_{S}(t)\right)} \\
& A^{S N c}=s_{\min }^{S N c} * e^{\left(c d_{s n c} * D A_{S}(t)\right)}
\end{aligned}
$$

where, $s_{\max }^{S T N}, s_{\min }^{G P e}$, and $s_{\min }^{S N C}$ are lateral connection strengths at the basal spontaneous activity of the population without any external dopaminergic influence in $S T N, G P e$, and $S N c$, respectively. $c d_{s t n}, c d_{g p e}$, and $c d_{s n c}$ were the factors by which DA affects the lateral connections in $S T N, G P e$, and $S N c$ populations respectively, $D A_{S}(t)$ is the instantaneous DA level, which is the spatial average DA concentration of all the terminals. All parameter values are given in Supplementary material-4.

The post-synaptic effect of DA in SNc, STN and GPe was modelled similar to (Muddapu et al., 2019),

$$
W_{x \rightarrow y}=\left(1-c d 2 * D A_{s}(t)\right) * w_{x \rightarrow y}
$$

where, $\quad w_{x \rightarrow y}$ is the synaptic weight $\quad(S T N \rightarrow G P e, G P e \rightarrow S T N, S T N \rightarrow S T N, G P e \rightarrow$ $G P e, S T N \rightarrow S N c, S N c \rightarrow S N c, M S N \rightarrow S N c$ ), $c d 2$ is the parameter that affects the postsynaptic current, $D A_{s}(t)$ is the instantaneous DA level.

The effect of DA in the MSN population occurs on both synaptic and intrinsic ion channels (Surmeier et al., 2007). The cortical inputs to MSN were modulated by DA as similar to (Humphries et al., 2009),

$$
I_{D A}^{x}(t)=I_{C T X \rightarrow M S N}^{x}(t) *\left(1+\left(\frac{\beta_{D A}}{\alpha_{D A}^{y}}\right) * D A_{S}(t)\right)
$$

where, $I_{C T X \rightarrow M S N}^{x}$ is the synaptic current from $C T X$ to $M S N$ (where $x=N M D A$ or $A M P A$ ), $D A_{S}(t)$ is the instantaneous DA level, $\alpha_{D A}^{y}$ is the DA effect on $y$ neuron (where $y=D 1-$ $M S N(G S)$ or D1 - MSN (G)), $\beta_{D A}$ was adapted from (Humphries et al., 2009). 
In addition to modulating cortical afferent connections, DA also has effects on the intrinsic ion channels (Humphries et al., 2009) which was modelled in Izhikevich neuronal model as,

$$
\begin{aligned}
& v_{r}^{D A}=v_{r}^{M S N} *\left(1+K^{M S N} *\left(\frac{D A_{s}(t)}{\alpha_{D A}^{y}}\right)\right) \\
& d_{m s n}^{D A}=d_{m s n} *\left(1-L^{M S N} *\left(\frac{D A_{s}(t)}{\alpha_{D A}^{y}}\right)\right)
\end{aligned}
$$

where, $v_{r}^{D A}$ and $d_{m s n}^{D A}$ are the DA-modulated resting potential and after-spike reset value of $M S N$ respectively, $v_{r}^{M S N}$, and $d_{m s n}$ are the resting potentials and after-spike reset value of $M S N$ respectively, $D A_{S}(t)$ is the instantaneous DA level; $\alpha_{D A}^{y}$ is the DA effect on $y$ neuron (where $y=D 1-M S N(G S)$ or $D 1-M S N(G)$ ), $K^{M S N}$ and $L^{M S N}$ were adapted from (Humphries et al., 2009).

\subsubsection{Substance $P$ modulation:}

SP modulates excitatory afferent connections of SNc (soma) and D1 MSN (G) in the proposed LIT model (Figure 1). It was observed that SP modulates the glutamatergic afferents of MSNs directly (Blomeley and Bracci, 2008) or indirectly (Blomeley et al., 2009) by co-release of SP by GABAergic D1 MSNs (Buxton et al., 2017; Reiner et al., 2010). In the proposed LIT model, we modelled SP-modulation of glutamatergic afferents of the D1 MSN (G) population by the D1 MSN (GS) population similar to (Buxton et al., 2017). It was observed that SP and tachykinin NK1 receptor (NK1-R) are highly expressed within the SNc (Lessard and Pickel, 2005; Mantyh et al., 1984; Ribeiro-da-Silva and Hökfelt, 2000; Sutoo et al., 1999; Thornton and Vink, 2015). SP-containing striatal neurons project to dopaminergic neurons where SP potentiates the release of striatal DA (Brimblecombe and Cragg, 2015; Thornton and Vink, 2015). It was reported that DA-dependent decrease in SP levels was observed in the basal ganglia regions (Sivam, 1991; Thornton et al., 2010; Thornton and Vink, 2015). Therefore, there exists a feedback regulation between DA and SP, which helps in maintaining DA homeostasis (Thornton et al., 2010; Thornton and Vink, 2015). In the proposed LIT model, we assumed that SP modulates STN glutamatergic inputs to SNc such that increased SP levels lead to excitation of SNc, which, in turn, enhances striatal DA level, modelled similar to (Buxton et al., 2017). Also, we incorporated SP-DA feedback regulation (SDFR) in SP-modulation in the 
proposed LIT model. The SP-modulation of glutamatergic inputs to D1 MSN (G) and SNc along with SDFR was given as,

$$
\begin{gathered}
I_{i j}^{x \rightarrow y}(t)=W_{x \rightarrow y} * h_{i j}^{x \rightarrow y}(t) * N S P * S D F R *\left(v_{i j}^{y}(t)-E_{\text {Recep }}\right) \\
N S P=\left(1+w_{s p} * N_{i j}^{s p}\left(t-\tau_{d}^{s p}\right)\right) \\
N_{i j}^{s p}(t)=\beta_{s p} *\left[1-e^{\left.\left(\frac{-A_{i j}^{s p}(t)}{\lambda_{s p}}\right)^{b_{s p}}\right]}\right] \\
A_{i j}^{s p}(t)=\left[e^{\left(\frac{-s_{i j}^{x}(t)}{\tau_{f}^{s p}}\right)}-e^{\left.\left(\frac{-s_{i j}^{x}(t)}{\tau_{r}^{s p}}\right)\right]}\right. \\
S D F R=\left(1-D A_{s}(t)\right)
\end{gathered}
$$

where, $h_{i j}^{x \rightarrow y}$ is the gating variable for the synaptic current from $x$ to $y, W_{x \rightarrow y}$ is the synaptic weight from neuron $x$ to $y, S_{i j}^{x}$ is the spiking activity of neuron $x$ at time $t, v_{i j}^{y}$ is the membrane potential of the neuron $y$ for the neuron at the location $(i, j), E_{\text {Recep }}$ is the receptor-associated synaptic potential $($ Recep $=$ NMDA/AMPA $), \tau_{d}^{s p}$ is the fixed time delay between MSN activity and the onset of neuropeptide effect, $\beta_{s p}$ is the gain factor, $N_{i j}^{s p}$ is the modulatory effect of SP, $w_{S p}$ is the influence of SP on $w_{S T N \rightarrow S N C}, A_{i j}^{S p}$ is the amplitude of SP released which is induced by spiking activity $\left(S_{i j}^{x}\right), D A_{s}(t)$ is the instantaneous DA level, $b_{s p}$ and $\lambda_{s p}$ were adapted from (Buxton et al., 2017) and given in Supplementary material-4.

\subsection{Total Synaptic Current Received by Each Neuronal Type}

\subsubsection{SNc:}

The total synaptic current received by a $S N C$ neuron at the lattice position $(i, j)$ is the summation of the glutamatergic input from the STN neurons, considering both $N M D A$ and $A M P A$ receptor activation, comprising the GABAergic inputs from the $D 1-M S N(G S)$ and $D 1-M S N(G)$ neurons and lateral GABAergic current from other SNC neurons. 


$$
\begin{aligned}
I_{i j}^{S N c S y n}=F_{S T N} & \rightarrow S N c *\left(I_{i j}^{N M D A \rightarrow S N C}+I_{i j}^{A M P A \rightarrow S N C}\right) \\
& +\left(F_{D 1-M S N}(G) \rightarrow S N c * I_{i j}^{D 1-M S N(G) \rightarrow S N c}\right) \\
& +\left(F_{D 1-M S N}(G S) \rightarrow S N C * I_{i j}^{D 1-M S N(G S) \rightarrow S N C}\right)+I_{i j}^{G A B A l a t}
\end{aligned}
$$

where, $I_{i j}^{N M D A \rightarrow S N C}$ and $I_{i j}^{A M P A \rightarrow S N C}$ are the glutamatergic currents corresponding to $N M D A$ and $A M P A$ receptors activation respectively; $I_{i j}^{D 1-M S N(G) \rightarrow S N C}$ and $I_{i j}^{D 1-M S N(G S) \rightarrow S N C}$ are the GABAergic inputs from the $D 1-M S N(G)$ and $D 1-M S N(G S)$ neurons respectively; $I_{i j}^{\text {GABAlat }}$ is the lateral GABAergic current from other $S N C$ neurons; $F_{S T N \rightarrow S N C}$ is the scaling factor for the glutamatergic current from STN neuron; $F_{D 1-M S N}(G) \rightarrow S N C$ is the scaling factor for the GABAergic current from $D 1-M S N(G)$ neuron; $F_{D 1-M S N}(G S) \rightarrow S N c$ is the scaling factor for the GABAergic current from D1 - MSN (GS) neuron.

\subsubsection{GPe:}

The total synaptic current received by a GPe neuron at the lattice position $(i, j)$ is the summation of the glutamatergic input from the STN neurons considering both NMDA and $A M P A$ receptors activation and the lateral GABAergic current from other GPe neurons.

$$
I_{i j}^{G P e s y n}=I_{i j}^{N M D A \rightarrow G P e}+I_{i j}^{A M P A \rightarrow G P e}+I_{i j}^{G A B A l a t}
$$

where, $I_{i j}^{N M D A \rightarrow G P e}$ and $I_{i j}^{A M P A \rightarrow G P e}$ are the glutamatergic currents from STN neuron considering both $N M D A$ and $A M P A$ receptors activation respectively; $I_{i j}^{G A B A l a t}$ is the lateral GABAergic current from other GPe neurons.

\subsubsection{STN:}

The total synaptic current received by a $S T N$ neuron at the lattice position $(i, j)$ is the summation of the GABAergic input from the GPe neurons and the lateral glutamatergic input from other STN neurons considering both NMDA and AMPA receptors activation.

$$
I_{i j}^{S T N s y n}=I_{i j}^{G A B A \rightarrow S T N}+I_{i j}^{N M D A l a t}+I_{i j}^{A M P A l a t}
$$

where, $I_{i j}^{G A B A \rightarrow S T N}$ is the GABAergic current from GPe neuron; $I_{i j}^{N M D A l a t}$ and $I_{i j}^{A M P A l a t}$ are the lateral glutamatergic current from other $S T N$ neurons considering both $N M D A$ and $A M P A$ receptors activation, respectively. 


\subsubsection{D1-MSN (GS):}

The total synaptic current received by a $D 1-M S N(G S)$ neuron at the lattice position $(i, j)$ is the summation of the GABAergic input from the $D 1-M S N(G)$ neurons and the glutamatergic input from CTX neurons considering both $N M D A$ and $A M P A$ receptors activation.

$$
\begin{aligned}
I_{i j}^{D 1-M S N(G S) s y n} & \\
& =I_{i j}^{G A B A \rightarrow D 1-M S N(G S)}+I_{i j}^{N M D A \rightarrow D 1-M S N(G S)} \\
& +I_{i j}^{A M P A \rightarrow D 1-M S N(G S)}
\end{aligned}
$$

where, $I_{i j}^{G A B A \rightarrow D 1-M S N(G S)}$ is the GABAergic current from $D 1-M S N(G)$ neuron, $I_{i j}^{N M D A \rightarrow D 1-M S N(G S)}$, and $I_{i j}^{A M P A \rightarrow D 1-M S N(G S)}$ are the glutamatergic current from CTX neurons considering both $N M D A$ and $A M P A$ receptors activation, respectively.

\subsubsection{D1-MSN (G):}

The total synaptic current received by a $D 1-M S N(G)$ neuron at the lattice position $(i, j)$ is the summation of the GABAergic input from the $D 1-M S N(G S)$ neurons and the glutamatergic input from $C T X$ neurons considering both $N M D A$ and $A M P A$ receptor activation.

$$
\begin{aligned}
I_{i j}^{D 1-M S N(G) S y n} & =I_{i j}^{G A B A \rightarrow D 1-M S N(G)}+I_{i j}^{N M D A \rightarrow D 1-M S N(G)} \\
& +I_{i j}^{A M P A \rightarrow D 1-M S N(G)}
\end{aligned}
$$

where, $I_{i j}^{G A B A \rightarrow D 1-M S N(G)}$ is the GABAergic current from $D 1-M S N(G S)$ neuron, $I_{i j}^{N M D A \rightarrow D 1-M S N(G)}$, and $I_{i j}^{A M P A \rightarrow D 1-M S N(G)}$ are the glutamatergic current from CTX neurons considering both $N M D A$ and $A M P A$ receptors activation, respectively.

\subsection{Neurodegeneration of SNc neurons}

Calcium plays a dual role in living organisms as a survival factor or a ruthless killer (Orrenius et al., 2003). For the survival of neurons, minimal (physiological) levels of glutamate stimulation are required. Under normal conditions, calcium concentration within a cell is tightly regulated by pumps, transporters, calcium-binding proteins, endoplasmic reticulum (ER) and mitochondria (Surmeier et al., 2011; Wojda et al., 2008). Due to prolonged calcium 
influx driven by excitotoxicity, the calcium homeostasis within the cell is disturbed, which results in cellular imbalance, leading to activation of apoptotic pathways (Bano and Ankarcrona, 2018). The SNc soma undergoes degeneration when calcium builds up inside the cell becomes high, resulting in calcium loading inside ER and mitochondria, which leads to ER-stress-induced and mitochondrial-induced apoptosis respectively (Malhotra and Kaufman, 2011). In the proposed LIT model, we incorporate a mechanism of programmed cell death, whereby a SNc neuron under high stress (high calcium levels) kills itself. The stress in a given SNc neuron was observed by monitoring the intracellular calcium concentrations in the cytoplasm, ER, and mitochondria.

The SNc neuron undergoes ER-stress-induced apoptosis when calcium levels in ER cross a certain threshold $\left(E R_{\text {thres }}\right)$. Under such conditions, the particular SNc neuron gets eliminated as follows,

$$
\text { if } C a_{i j}^{E R}(t)>E R_{\text {thres }} \text {, then } v_{i j}^{S N c}(t)=0
$$

where, $C a_{i j}^{E R}$ is the calcium concentration in the ER, $E R_{\text {thres }}$ is the calcium concentration threshold after which ER-stress induced apoptosis gets initiated, $v_{i j}^{S N c}$ is the membrane voltage of neuron at the lattice position $(i, j)$.

The SNc neuron undergoes mitochondria-induced apoptosis when calcium levels in mitochondria cross a certain threshold $\left(M T_{\text {thres }}\right)$. Then that particular $\mathrm{SNc}$ neuron will be eliminated as follows,

$$
\text { if } \quad C a_{i j}^{M T}(t)>M T_{\text {thres }} \text {, then } v_{i j}^{S N c}(t)=0
$$

where, $C a_{i j}^{M T}$ is the calcium concentration in mitochondria, $M T_{\text {thres }}$ is the calcium concentration threshold after which mitochondria-induced apoptosis gets initiated, $v_{i j}^{S N C}$ is the membrane voltage of neuron at the lattice position $(i, j)$.

When calcium concentration in ER crosses a certain threshold, there is an efflux of excess calcium from ER out into cytoplasm, which in turn activates calpain, resulting in activation of proapoptotic factors through cytochrome-c independent apoptotic pathway. Similarly, when calcium concentration in MT crosses a certain threshold, excess calcium in MT results in the formation of mitochondrial transition pores (MTPs). Proapoptotic cytochrome-c released from MT through MTPs, which triggers cytochrome-c dependent 
apoptosis. In the proposed modeling study, when the apoptotic signal gets activated from either of the pathways in a particular neuron, we formulate an approach wherein that particular neuron was eliminated by making $v_{i j}^{S N C}(t)=0$ from the time $t$ till the end of the simulation.

\subsection{Terminal degeneration of SNc neurons}

DA is the primary contributor to the oxidative stress in the neuron (Lotharius et al., 2005; Luo and Roth, 2000; Miyazaki and Asanuma, 2008). To evade oxidative stress, SNc neurons tightly regulate the DA turnover processes (Guo et al., 2018). It was suggested that methamphetamineinduced dopaminergic nerve terminal loss (Ares-Santos et al., 2014; Cadet et al., 2003; Ricaurte et al., 1982, 1984) is precipitated by oxidative stress (De Vito and Wagner, 1989) by enhancing cytoplasmic DA levels (Larsen et al., 2002; Mark et al., 2004). In the proposed LIT model, the oxidative stress in the SNc terminals was observed by monitoring intracellular reactive oxygen species (ROS) concentration. The SNc terminal is eliminated when ROS levels in the terminal cross certain threshold $\left(R O S_{\text {thres }}\right)$ as follows,

$$
\text { if } \operatorname{ROS}_{i j}^{T}(t)>R_{\text {thres }}, \text { then } C a_{i j}^{T}(t)=0
$$

where, $R O S_{i j}^{T}$ is the ROS concentration in the SNc terminal, $R O S_{\text {thres }}$ is the ROS concentration threshold above which oxidative stress-induced terminal degeneration gets initiated; $C a_{i j}^{T}$ is the calcium concentration of the SNc terminal at the lattice position $(i, j)$.

When ROS level crosses a certain threshold, excess ROS triggers degeneration of the terminal. In the proposed modeling study, when ROS level crosses the threshold in a particular terminal, we formulate an approach wherein that particular terminal was eliminated by making $C a_{i j}^{T}(t)=0$ from the time $t$ till the end of the simulation since calcium plays an important role in terminal functioning.

\subsection{Neuroprotective Strategies}

\subsubsection{Levodopa Therapy}

To alleviate PD symptoms, the most potent drug, LDOPA, a precursor of DA, is typically administrated (Jankovic and Aguilar, 2008). During medication, serum LDOPA is taken up from the blood into the extracellular fluid by aromatic L-amino acid transporter by competing with other amino acids (Camargo et al., 2014; Figura et al., 2018). L-DOPA, thus absorbed into the bloodstream, later enters SNc terminals and gets converted to DA by aromatic L-amino 
acid decarboxylase (Khor and Hsu, 2007). In the proposed LIT model, serum LDOPA uptake into SNc terminal from the blood was modelled as a single step along with competition with other amino acids such as tyrosine and tryptophan (Porenta and Riederer, 1982). It was represented using the Michaelis-Menten equation (Chou, 1976) where serum LDOPA competes with serum tyrosine and serum tryptophan for transporter (Reed et al., 2012) as given below:

$$
V_{\text {trans }}=\frac{V_{\text {trans }}^{\max } *\left[L D O P A_{S}\right]}{K_{m}^{L D O P A_{s}} *\left(1+\frac{\left[T Y R_{S}\right]}{K_{a}^{T Y R_{S}}}+\frac{\left[T R P_{S}\right]}{K_{a}^{T R P P_{s}}}\right)+\left[L D O P A_{S}\right]}
$$

where, $V_{\text {trans }}^{\max }$ is the maximum flux through aromatic L-amino acid transporter, $\left[L D O P A_{S}\right]$ is the serum LDOPA concentration, $K_{m}^{L D O P A_{S}}$ is the concentration of $\left[L D O P A_{S}\right]$ at which velocity of the transporter attained half of the maximal velocity, $\left[T Y R_{S}\right]$ is the serum tyrosine concentration, $\left[T R P_{S}\right]$ is the serum tryptophan concentration. $K_{a}^{T Y R_{S}}$ is the affinity constant for $\left[T Y R_{S}\right], K_{a}^{T R P_{s}}$ is the affinity constant for $\left[T R P_{S}\right]$.

LDOPA therapy was implemented in the proposed LIT model by the following criterion,

$$
\begin{gathered}
{\left[L D O P A_{s}\right]\left(N_{s c}^{Z}, t\right)=\left\{\begin{array}{rr}
0, & N_{s c}^{Z}(t)>T_{l}^{Z} \\
{\left[L D O P A_{s}^{\text {med }}\right],} & N_{s c}^{Z}(t) \leq T_{l}^{Z}
\end{array}\right.} \\
T_{l}^{Z}=P_{z}^{s n c}-\left(p c l * P_{z}^{s n c}\right)
\end{gathered}
$$

where, $\left[L D O P A_{s}\right]\left(N_{S c}^{Z}, t\right)$ is the instantaneous serum $[L D O P A]$ concentration based on the number of surviving SNc neurons or terminals at the time $(t)\left(N_{S c}^{Z}(t)\right),\left[L D O P A_{s}^{\text {med }}\right]$ is the serum $[L D O P A]$ concentration during medication, $N_{S c}^{Z}(t)$ is the instantaneous number of surviving SNc neurons or terminals, $p c l$ is the percentage of SNc cell or terminal loss (25\%) at which therapeutic intervention was employed $(p c l=0.25), T_{l}^{Z}$ represents the number of surviving SNc cells or terminals at which therapeutic intervention was employed, $P_{Z}^{s n c}$ is the population size of $z$ ( $z=$ soma or terminal). In the present study, the therapeutic intervention is given at $25 \% \mathrm{SNc}$ cell or terminal loss. 


\subsubsection{SP antagonist therapy}

It was reported that SP exacerbated dopaminergic neurodegeneration in mice (Wang et al., 2014), and therefore administrating SP antagonists creates neuroprotection of dopaminergic neurons in PD (Johnson et al., 2017; Thornton and Vink, 2012, 2015). In the proposed LIT model, SP antagonist effect was implemented as,

$$
w_{s p a}=w_{s p} * \delta_{s p a}
$$

where, $w_{s p}$ is the influence of SP on $w_{S T N \rightarrow S N C}, \delta_{s p a}$ is the proportion of SP inhibition, $w_{s p a}$ is the influence of SP on $w_{S T N \rightarrow S N C}$ under SP antagonist therapy.

The SP antagonist therapy was implemented in the proposed LIT model by the following criterion,

$$
\delta_{s p a}\left(N_{s c}^{Z}, t\right)=\left\{\begin{aligned}
0, & N_{s c}^{Z}(t)>T_{l}^{Z} \\
\delta_{s p a}^{\text {med }}, & N_{s c}^{Z}(t) \leq T_{l}^{Z}
\end{aligned}\right.
$$

where, $\delta_{\text {spa }}\left(N_{s c}^{Z}, t\right)$ is the instantaneous proportion of SP inhibition based on the number of surviving SNc neurons or terminals at the time $(t)\left(N_{s c}^{Z}(t)\right), \delta_{s p a}^{\text {med }}$ is the proportion of SP inhibition during therapy, $N_{s c}^{Z}(t)$ is the instantaneous number of surviving SNc neurons or terminals, $T_{l}^{Z}$ represents the number of surviving $\mathrm{SNc}$ cells or terminals at which therapeutic intervention was employed ( $z=$ soma or terminal).

\subsubsection{Glutathione therapy}

The impaired DA metabolism causes oxidative stress (ROS), which in turn leads to PD pathogenesis (Masato et al., 2019). It was reported that abnormal activity of vesicular monoamine transporter 2 (VMAT2) leads to reduced vesicular DA storage and increased cytoplasmic DA which results in oxidative stress-induced degeneration of cell bodies (soma) and terminals (Caudle et al., 2007; Kariya et al., 2005; Mingazov and Ugryumov, 2019; Pifl et al., 2014). It was reported that the glutathione (GSH) administration results in the improvement of PD symptoms, but the underlying mechanism is not clear (Hauser et al., 2009; Mischley et al., 2017; Zeevalk et al., 2008). We suggest that glutathione administration might result in 
scavenging of ROS, which in turn leads to neuroprotection (Li et al., 2015). In the proposed LIT model, glutathione effect was implemented as,

$$
\left[G S H_{g s t}^{z}\right]=\left[G S H^{z}\right]+\left[G S H_{g S}^{z}\right]
$$

where, $\left[G_{S S H}^{z}\right]$ is the GSH concentration under glutathione therapy $(z=$ soma or terminal), $\left[G S H^{z}\right]$ is the GSH concentration.

The glutathione therapy was implemented in the proposed LIT model by the following criterion,

$$
\left[G S H_{g s}^{z}\right]\left(N_{s c}^{Z}, t\right)=\left\{\begin{aligned}
0, & N_{s c}^{Z}(t)>T_{l}^{Z} \\
{\left[G S H_{m e d}^{Z}\right], } & N_{s c}^{Z}(t) \leq T_{l}^{Z}
\end{aligned}\right.
$$

where, $\left[G S H_{g s}^{z}\right]\left(N_{s c}^{Z}, t\right)$ is the instantaneous $[G S H]$ therapy based on the number of surviving SNc neurons or terminals at the time $(t)\left(N_{s c}^{Z}(t)\right), N_{s c}^{Z}(t)$ is the instantaneous number of surviving SNc neurons or terminals, $\left[G S H_{\text {med }}^{Z}\right]$ is the $[G S H]$ concentration dosage under $G S H$ therapy, $T_{l}^{Z}$ represents the number of surviving SNc cells or terminals at which therapeutic intervention was employed ( $z=$ soma or terminal).

\section{RESULTS}

We investigate the Izhikevich models of the neurons of CTX, MSN, GPe, and STN, which were chosen from the literature (Humphries et al., 2009; Mandali et al., 2015; Michmizos and Nikita, 2011) for their characteristic firing pattern and other biological properties (Figure 2). Along with the above Izhikevich neuronal models, we also investigate the biophysical neuronal model of SNc for its characteristic responses (Figure 3). Next, we explored the effect of DA and SP on the network of MSN and SNc neurons and compared them with published data (Figure 4).

Then, we show the effect of homogeneous (Figure 5) and heterogeneous (Figure 6) energy deficit conditions on the progression of SNc somas and terminals loss. Next, we show the effect of extracellular LDOPA on the progression of SNc soma and terminal loss under energy deficit conditions (Figure 7A). Finally, we explore various therapeutics such as LDOPA 
(Figure 7B), SP antagonist (Figure 7C), and glutathione (Figure 7D) for their neuroprotective effect on the progression of SNc somas and terminals loss under energy deficit conditions.

\subsection{Characteristic Firing Response of Different Neuronal Types}

The response of a single neuronal model of five different neuronal types involved in the proposed LIT model for different external applied currents is shown in Figure 2. The basal firing frequencies of the different neuronal types were matched with experimentally observed firing (Tripathy et al., 2014) by adjusting $I_{i j}^{x}$ parameter given in Supplementary material-2.

The pyramidal neurons in the cortex are broadly classified into three types, namely regular spiking (RS), intrinsic (inactivating) bursting, and non-inactivating bursting neurons. The regular spiking neurons further subdivided into fast-adapting and slow-adapting types of neurons (Degenetais, 2002). The time-averaged firing rate of all neuronal types varies widely, ranging from $<1 \mathrm{~Hz}$ up to several tens of hertz (Griffith and Horn, 1966; Koch and Fuster, 1989). The spontaneous firing rates of all pyramidal cortical neuronal types are as follows: fastadapting RS $(0.62 \pm 0.75 \mathrm{~Hz})$, slow-adapting RS $(0.90 \pm 1.23 \mathrm{~Hz})$, intrinsic bursting $(3.1 \pm 2.6 \mathrm{~Hz})$ and non-inactivating bursting $(2.8 \pm 3.2 \mathrm{~Hz})$ (Degenetais, 2002). In the proposed LIT model, we adjust $I_{i j}^{x}$ value such that the combined spontaneous and stimulusdriven firing rate of the pyramidal cortical neuron falls in the range of $10-15 \mathrm{~Hz}$ (Figure 2A).

The medium spiny neurons in the striatum broadly classified into two types based on the type of DA receptor present, namely D1 and D2-types. In the proposed LIT model, we consider only D1-type MSNs because they only project GABAergic inputs to SNc neurons (Gerfen, 1985). The spontaneous firing rate of MSN was observed experimentally to be in the range of $0.6-16.1 \mathrm{~Hz}$ (Mahon et al., 2006; Pitcher et al., 2007). In the proposed LIT model, we adjust $I_{i j}^{x}$ value such that the combined spontaneous and cortical-driven firing rate of MSN falls in the range of $10-20 \mathrm{~Hz}$ (Figure 2B).

The STN neurons exhibit two distinct types of firing patterns experimentally: tonic pacemaking firing and phasic high-frequency bursting (Allers et al., 2003; Beurrier et al., 1999). The spontaneous firing rate of STN neurons was observed experimentally to be in the range of $6-30 \mathrm{~Hz}$ (Allers et al., 2003; Lindahl et al., 2013). In the proposed LIT model, we adjust $I_{i j}^{x}$ value such that the STN spontaneous firing rate is $\sim 13 \mathrm{~Hz}$ (Figure 2D). 
bioRxiv preprint doi: https://doi.org/10.1101/2020.04.05.026807 this version posted April 6 , 2020. The copyright holder for this preprint (which was not certified by peer review) is the author/funder, who has granted bioRxiv a license to display the preprint in perpetuity. It is made available under aCC-BY-NC-ND 4.0 International license.

The GPe neurons exhibit an atypical type of firing pattern where bursts and pauses appear aperiodically in a continuous tonic high-frequency firing (Hegeman et al., 2016; Kita and Kita, 2011). The spontaneous firing rate of GPe neuron was observed experimentally to be in the range of $8-60 \mathrm{~Hz}$ (Bugaysen et al., 2010; Elias et al., 2008; Lindahl et al., 2013). In the proposed LIT model, we adjusted $I_{i j}^{x}$ value such that the GPe spontaneous firing rate was $\sim 30 \mathrm{~Hz}$ (Figure 2C).

A)
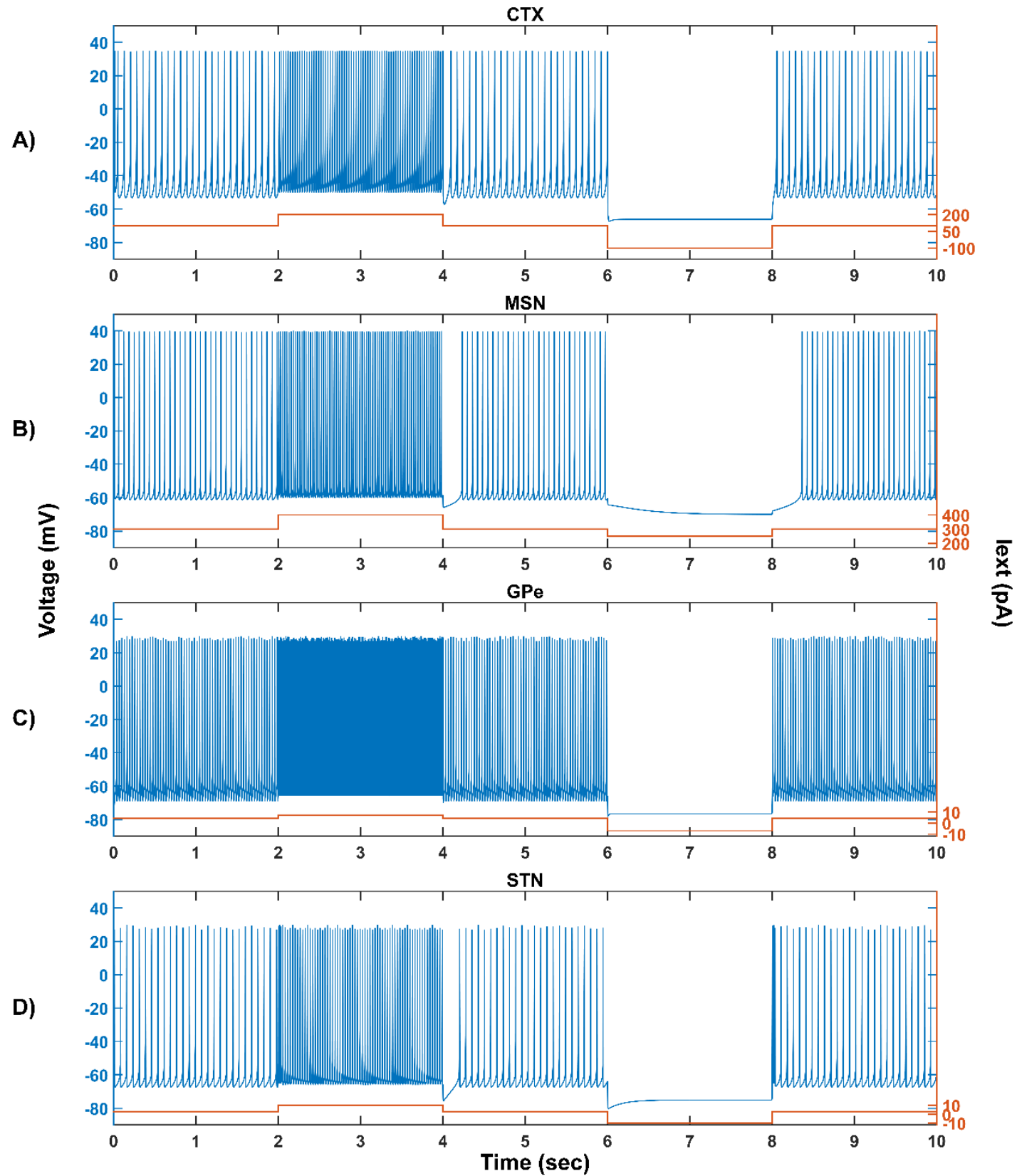

Figure 2: Characteristic behavior of individual neurons. Characteristic behavior of individual CTX (A), MSN (B), GPe (C), and STN (D) neuronal types. CTX, cortex; MSN, medium spiny neuron; GPe, globus pallidus externa; STN, subthalamic nucleus, lext, external current; $p A$, picoampere; $m V$, millivolt; sec, second. 
The SNc neurons exhibit two distinct types of firing patterns experimentally, namely background or low-frequency irregular tonic firing $(3-8 \mathrm{~Hz})$ and bursting or high-frequency regular phasic firing $(\sim 20 \mathrm{~Hz})$ (Grace and Bunney, 1984b, 1984a). In the proposed LIT model, SNc neurons spontaneously fire with a firing rate of $\sim 4 \mathrm{~Hz}$ (Figure 3). The calcium concentration inside the SNc neuron during the resting state was $\sim 1 \times 10^{-4} \mathrm{mM}$, and it rises up to $1 \times 10^{-3} \mathrm{mM}$ upon arrival of the action potential (Figure 3C) (Ben-Jonathan and Hnasko, 2001; Dedman and Kaetzel, 1997; Wojda et al., 2008). The dopamine released by SNc neuron during tonic spiking was $\sim 45 \times 10^{-6} \mathrm{mM}$, which was in the range of $(34-48) \times 10^{-6} \mathrm{mM}$ observed experimentally (Figure 3D) (Garris et al., 1997). Upon injection of depolarizing external current (continuous pulse $\left(I_{\text {ext }}=25 \times 10^{-6} p A\right)$ and duration $(1 \mathrm{sec})$ ), SNc neuron exhibits bursting type of firing which lasts for more than one second after the pulse was removed (Figure 2B, positive current), demonstrating the slow-adapting nature of SNc neuron due to excess calcium build-up inside the neuron (Figure 3C, positive current) (Kuznetsova et al., 2010). During the depolarizing current stimulation, SNc neurons exhibit the property within a burst that spikes after an initial spike showed a decrease in amplitude (Figure 3B, positive current), which is a characteristic bursting property of SNc neurons (Grace and Bunney, 1984a). The dopamine concentration released by SNc neuron during phasic bursting peaked at $\sim 118 \times 10^{-6} \mathrm{mM}$ (Figure 3D, positive current), which is in the range of (90220) $x 10^{-6} \mathrm{mM}$ (Chen and Budygin, 2007). Further increase in depolarizing current amplitude increases extracellular DA release exponentially but never exceeds beyond $1 \times 10^{-3} \mathrm{mM}$ (not shown) (Gonon, 1988). Upon injection of hyperpolarized external current (continuous pulse $\left(I_{\text {ext }}=-300 \times 10^{-6} \mathrm{pA}\right.$ ) and duration (1 sec)), SNc neuron exhibits quiescent state until stimulation was removed (Figure 3B, negative current). Due to hyperpolarized current stimulation, the calcium oscillation in SNc neuron was minimal (Figure $3 \mathrm{C}$, negative current), which was reflected in the near absence of extracellular DA release (Figure 3D, negative current).

The lateral connections in SNc, STN, and GPe neuronal populations were studied in the previous work (Muddapu et al., 2019). No lateral connections were considered in CTX and MSN neuronal populations for the simplification of the proposed LIT model.

\subsection{Neuromodulatory Effect of Dopamine on MSN and SNc Neuronal Populations}

In the MSN population, DA affects both synaptic and intrinsic ion channels (Surmeier et al., 2007). As the DA levels increase, the influence of cortical glutamatergic inputs on D1-type 
MSN increases resulting in monotonously increasing firing frequency (Figure 4A), which was consistent with experimental work (Cepeda et al., 1993) and previous modeling studies (Humphries et al., 2009). In the MSN population, SP affects synaptic ion channels, especially glutamatergic afferents (Blomeley et al., 2009; Blomeley and Bracci, 2008). As the SP levels (or SP scaling factor $\left(W_{s p}\right)$ ) increases, the influence of cortical glutamatergic inputs on D1type MSN increases resulting in monotonously increasing firing frequency (Figure 4B), which was similar to experimental (Blomeley and Bracci, 2008) and other modelling studies (Buxton et al., 2017).

A)

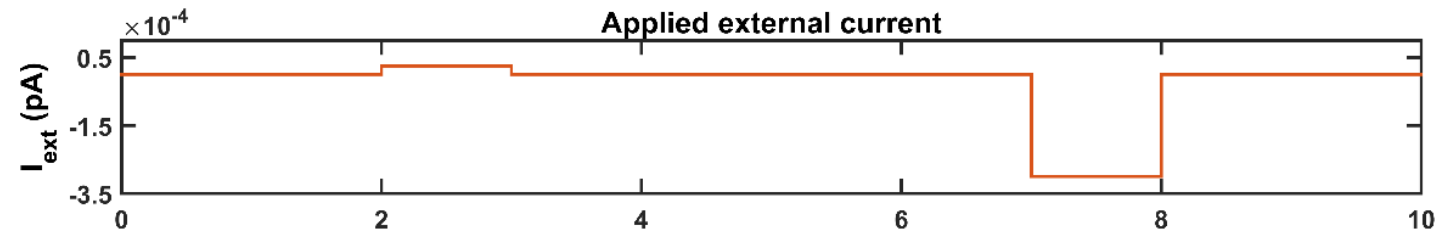

Membrane potential oscillations

B)

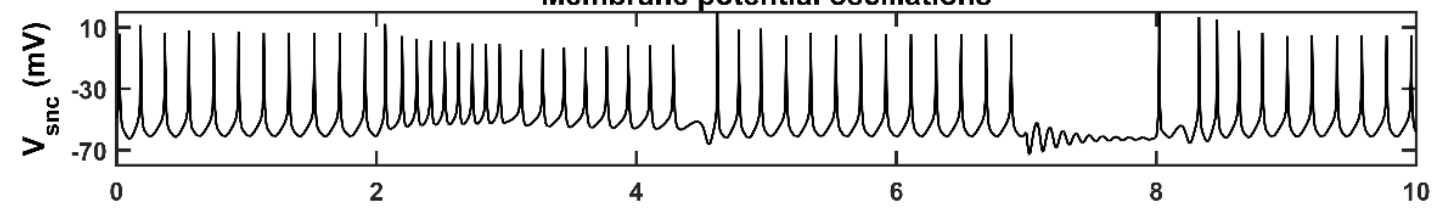

C)

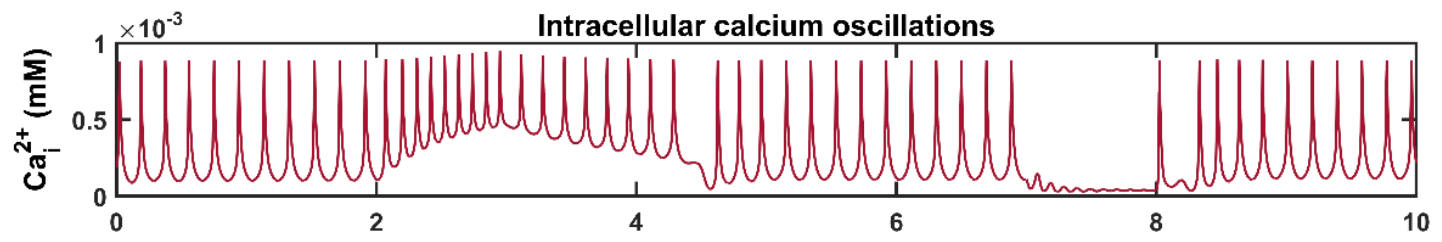

D)

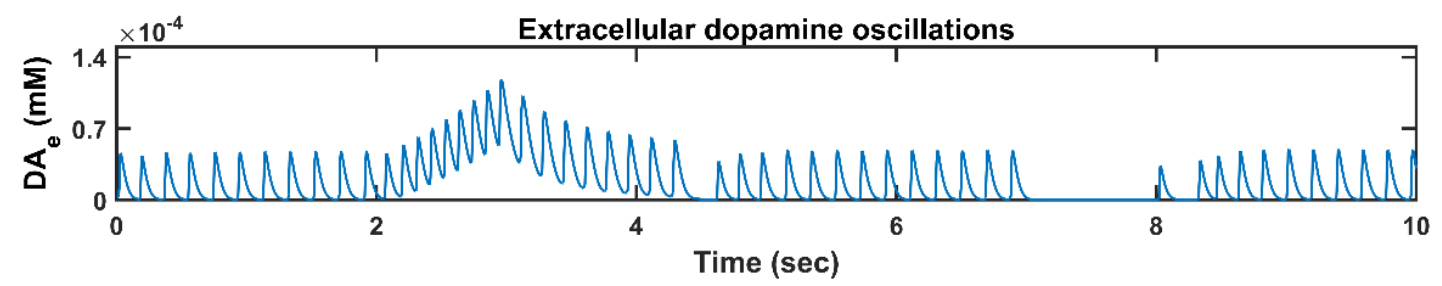

Figure 3: Characteristic behavior of individual SNc neuron. (A) Applied external current $\left(I_{\text {ext }}\right),(B)$ Membrane potential oscillations $\left(V_{s n c}\right)$, (C) Intracellular calcium oscillations $\left(\mathrm{Ca}_{i}^{2+}\right)$, (D) Extracellular dopamine concentration $\left(D A_{e}\right)$. $p A$, picoampere; $m V$, millivolt; sec, second; $m M$, millimolar.

In the SNc population, DA affects both synaptic and lateral connections (Muddapu et al., 2019). As the DA level increases, the influence of synaptic and lateral connection inputs on SNc increases resulting in non-monotonously decreasing firing frequency (Figure 4C) which was similar to experimental (Ford, 2014; Hebb and Robertson, 1999; Tepper and Lee, 2007; Vandecasteele et al., 2005) and other modelling studies (Muddapu et al., 2019). In the $\mathrm{SNc}$ population, SP affects the synaptic ion channels, especially glutamatergic afferents (Brimblecombe and Cragg, 2015; Thornton and Vink, 2015). As the SP level (or SP scaling factor $\left(W_{s p}\right)$ ) increases, the influence of STN glutamatergic inputs on SNc increases, resulting 
in monotonously increasing firing frequency (Figure 4D), which was similar to experimental studies (Brimblecombe and Cragg, 2015). The effect of DA on STN and GPe neuronal populations was simulated in the previous work (Muddapu et al., 2019).

A)

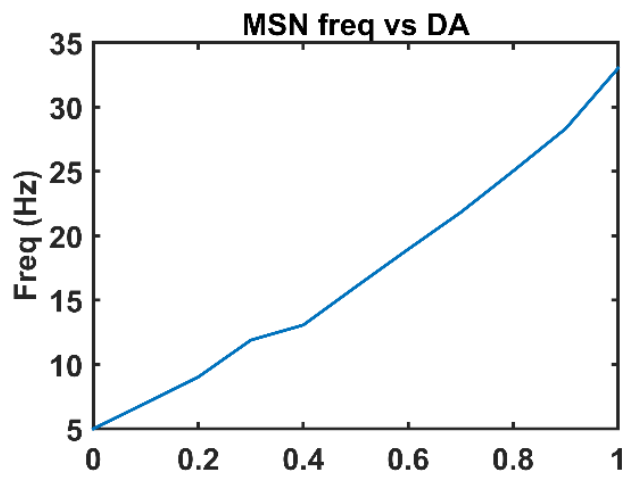

C)

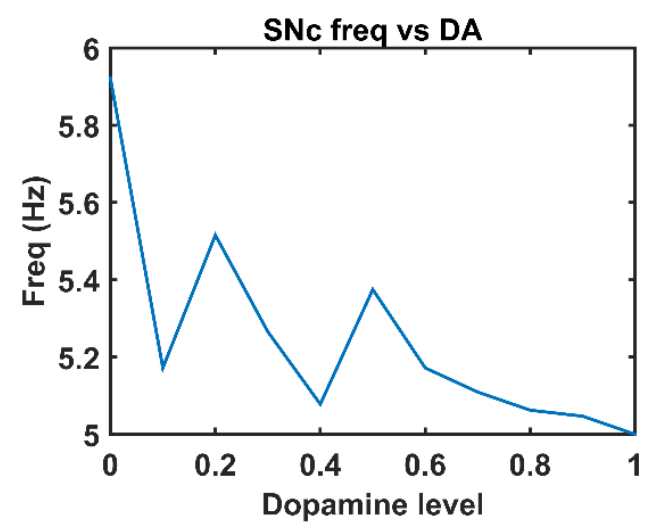

B)

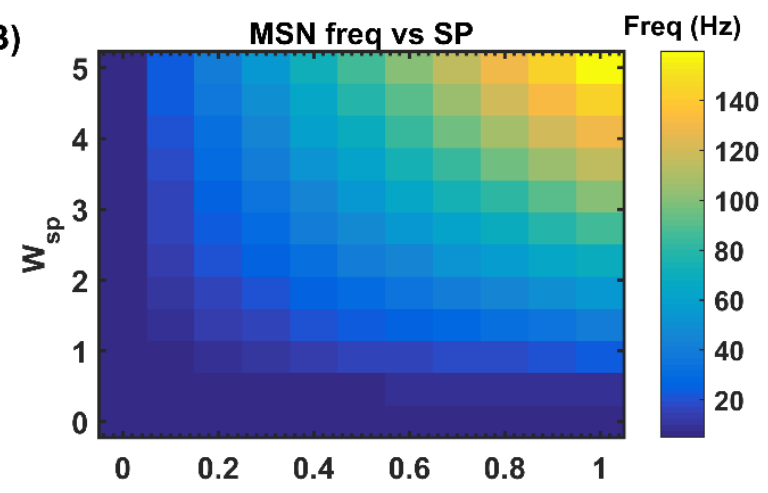

D)

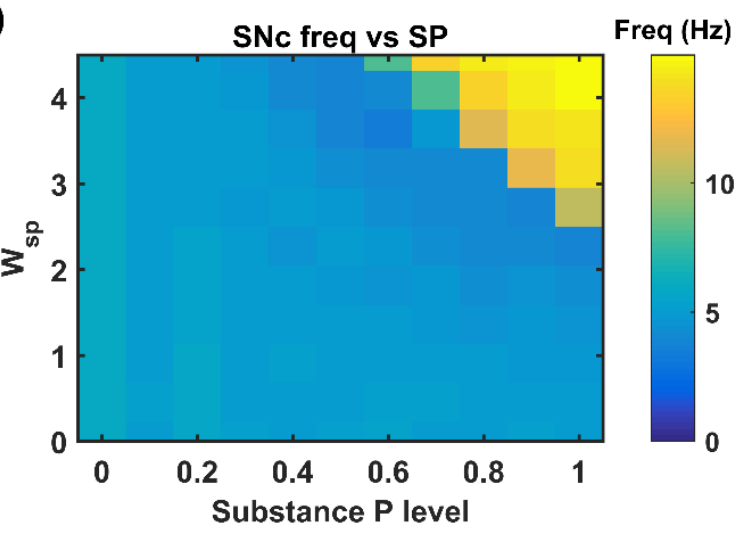

Figure 4: Neuromodulatory effects of DA and SP on MSN and SNc neuronal populations. Effect of dopamine on MSN (A) and SNC (C) populations. Effect of SP on MSN (B) and SNC (D). MSN, medium spiny neuron; $D A$, dopamine; Freq, frequency; $S P$, substance $P$; $S N c$, substantia nigra pars compacta; $W_{s p}$, scaling factor of SP influence; $\mathrm{Hz}$, hertz.

\subsection{Energy Deficiency Occurring Similarly in SNc Somas and Terminals}

In order to study energy deficiency as the possible root cause of SNc cell loss PD, we simulated ischemic conditions by modulating glucose and oxygen inputs in the proposed LIT model. As $\mathrm{SNc}$ somas and terminals are far from each other, the ischemic condition was implemented in two ways: homogeneous (energy deficiency occurs similarly in somas (midbrain) and terminals (striatum)) and heterogeneous (energy deficiency occurs differently in somas (midbrain) and terminals (striatum)). Homogeneous energy deficiency was implemented by reducing glucose and oxygen values by same proportions in both $\mathrm{SNc}$ somas and terminals. In the case of homogeneous energy deficiency, terminal loss starts with just $10 \%$ of somas and terminals in energy deficiency (Figure 5A, orange bar). However, soma loss starts at 70\% of somas and terminals in energy deficiency (Figure 5A, blue bar). To observe the influence of STN on SNc 
soma and terminal loss, the currents from STN to SNc were monitored, which showed higher positive currents after $60 \%$ of somas and terminals are in energy deficiency (Figure 5B). The higher positive current from STN also results in increased ROS production in both SNc somas and terminals (Figure 5C).
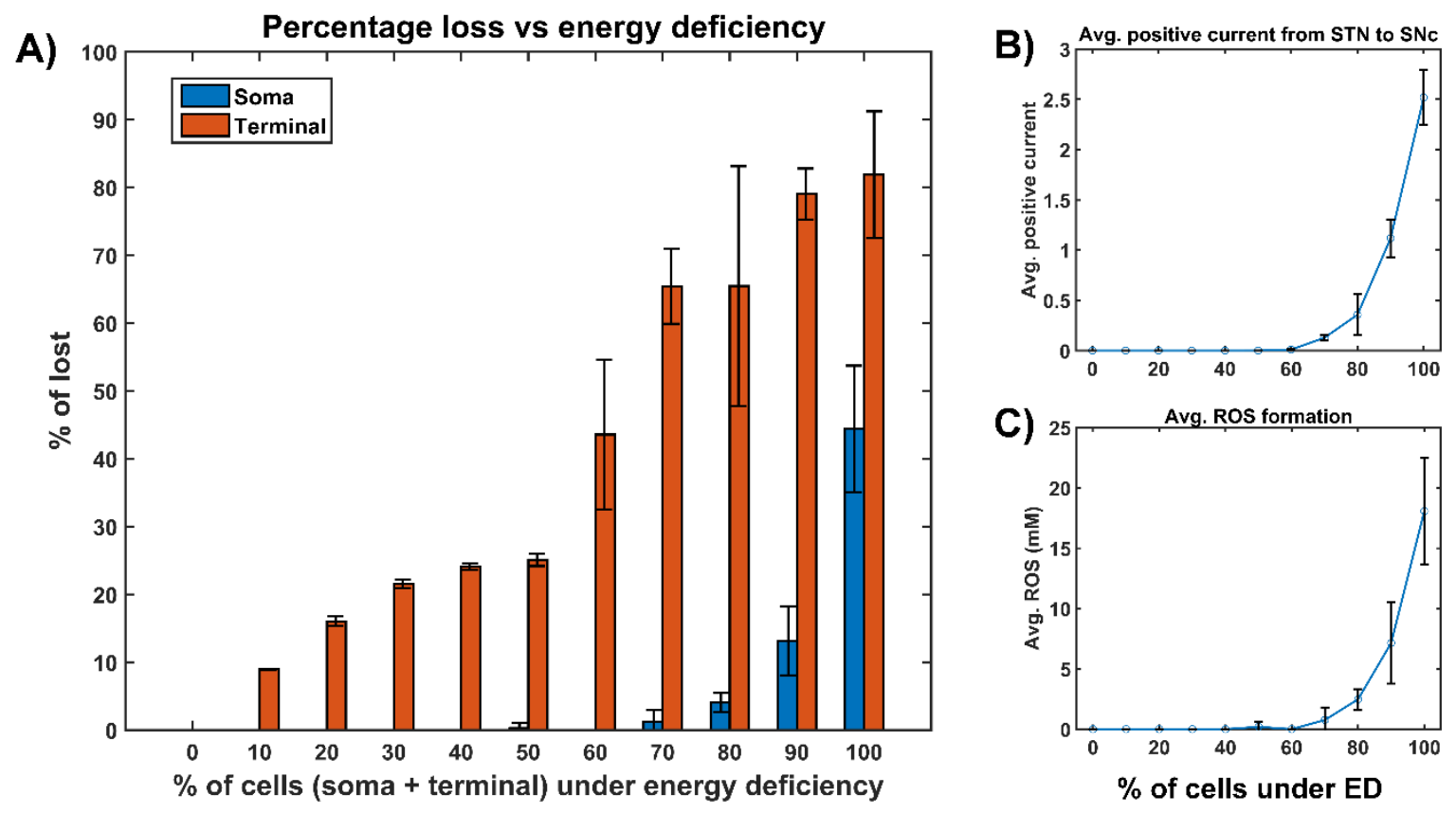

Figure 5: The model response under homogeneous energy deficiency. (A) The percentage loss of SNc somas and terminals, (B) Average positive current from STN to SNc, (C) Average ROS formation. STN, subthalamic nucleus; SNc, substantia nigra pars compacta; ROS, reactive oxygen species; $m M$, millimolar; $E D$, energy deficiency.
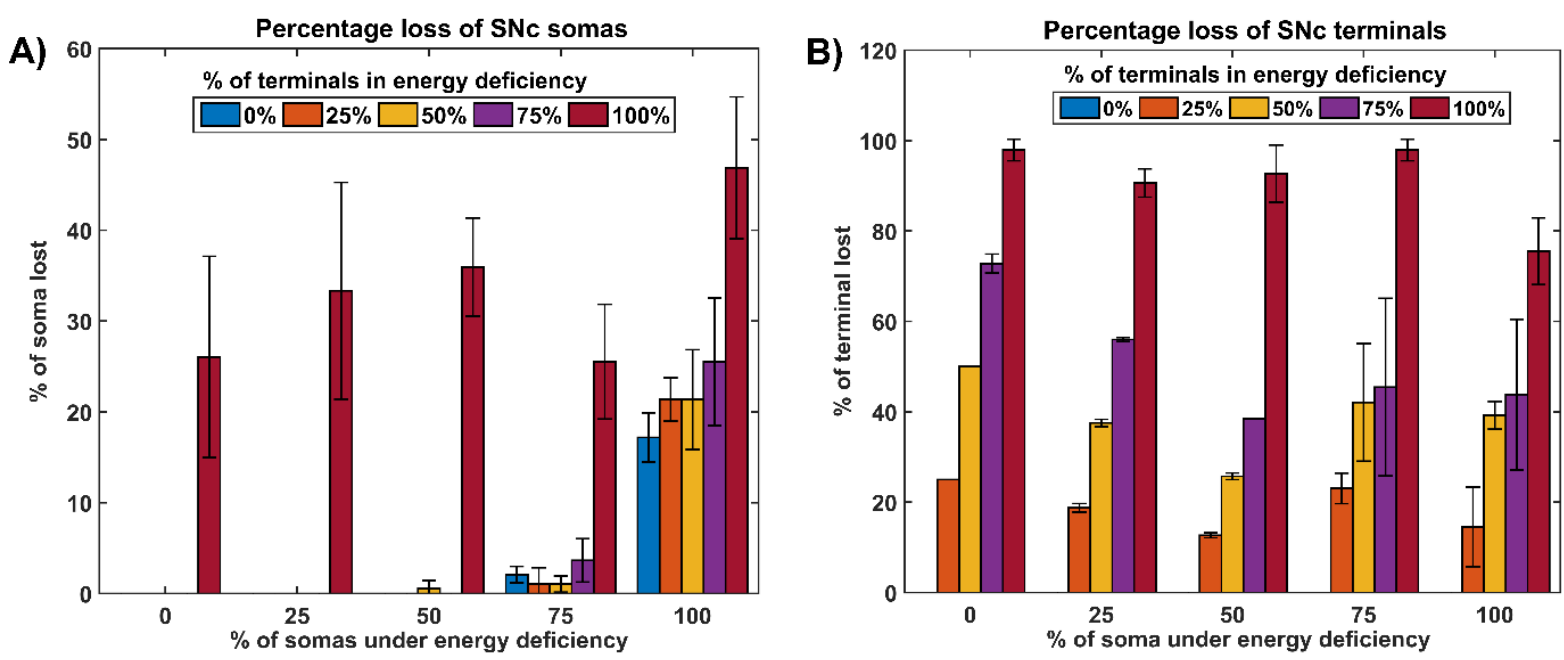

Figure 6: The model response under heterogeneous energy deficiency. (A) The percentage loss of SNc somas, (B) The percentage loss of SNc terminals. ED, energy deficiency; SNc, substantia nigra pars compacta.

\subsection{Energy Deficiency Occurring Differently in SNc Somas and Terminals}


Heterogeneous energy deficiency was implemented by reducing glucose and oxygen values by different proportions in both SNc somas and terminals. In the case of heterogeneous energy deficiency, the loss of somas was observed after $75 \%$ of somas are in energy deficiency for all energy deficiency levels in terminals (Figure 6A). The soma loss was minimal or non-existent when the percentage of somas in energy deficiency was below $75 \%$ for all levels of energy deficiency in terminals except $100 \%$. In the case when $100 \%$ of terminals are in energy deficiency, the loss of somas was above $20 \%$ for all levels of energy deficiency in somas, and maximum loss of somas ( 45\%) was observed when 100\% of somas are energy deficiency (Figure 6A). The terminal loss observed for all percentages of somas and terminals in energy deficiency except $0 \%$ of terminals in energy deficiency (Figure 6B). The terminal loss increases with an increase in the percentage of terminals in energy deficiency for all percentages of somas in energy deficiency (Figure 6B).

\subsection{Effect of Extracellular LDOPA}

In order to study the effect of extracellular (serum) LDOPA on SNc somas and terminal loss in energy deficiency (100\% energy deficiency), we have modified extracellular LDOPA concentration in the range from $36 \times 10^{-9} \mathrm{mM}$ to $36 \mathrm{mM}$ with the multiple of 10x (Figure 7A). With lower concentrations of extracellular LDOPA ranging from $36 \times 10^{-9} \mathrm{mM}$ to $36 \times 10^{-7} \mathrm{mM}$, a more significant loss ( 60\%) of SNc somas was observed when compared to SNc terminals ( 40\%) (Figure 7A). With higher concentrations of extracellular LDOPA ranging from $36 \times 10^{-4} \mathrm{mM}$ to $36 \mathrm{mM}$, more of SNc terminal loss ( 95\%) was observed when compared to SNc somas ( 35\%) (Figure 7B). At intermediate levels of extracellular LDOPA concentrations ranging from $36 \times 10^{-7} \mathrm{mM}$ to $36 \times 10^{-4} \mathrm{mM}$, the percentage loss of SNc somas and terminals were similar which was in the range of $50-60 \%$, and these are the extracellular LDOPA concentrations that were observed in previous studies (Cullen and WongLin, 2015; Khor and Hsu, 2007; Reed et al., 2012).

\subsection{Insights into the Mechanism of LDOPA-Induced Toxicity}

In order to test the hypothesis of LDOPA-induced toxicity, we have administered ranges of external LDOPA in the proposed model when the percentage loss of somas or terminals crosses $25 \%$ due to energy deficiency. When external LDOPA concentration $\left(36 \times 10^{-4} \mathrm{mM}\right)$ administered was near basal value, it was observed that the percentage loss of SNc somas and terminals was not altered much. When external LDOPA concentration administered was in the range from $36 \times 10^{-4} \mathrm{mM}$ to $36 \times 10^{-3} \mathrm{mM}$, it was observed that the percentage loss of SNc 
somas and terminals was decreasing. When external LDOPA concentration administered was above $36 \times 10^{-3} \mathrm{mM}$, it was observed that the percentage loss of SNc somas was similar, but the percentage loss of SNc terminals was higher when external LDOPA concentration was near basal value (Figure 7B).

From simulation results, it was observed that LDOPA indeed induced toxicity in SNc cells at higher concentrations, which might be due to excitotoxicity or oxidative stress or both. To evade LDOPA toxicity in all stages of LDOPA therapy in the case of PD, we need to understand the mechanism behind the toxicity. In order to do so, we co-administered two different drugs along with LDOPA, namely SP antagonists and glutathione, which led to target overexcitation in $\mathrm{SNc}$ somas (by reducing SP-mediated excitatory inputs to $\mathrm{SNc}$ ) and ROS build up in SNc terminals (by scavenging ROS) respectively. When SP antagonists are coadministered, it was observed that the percentage loss of SNc somas was decreasing with increasing inhibition of SP transmission (Figure 7C, blue bar). However, there was a change in the percentage loss of SNc terminals (Figure 7C, orange bar). When glutathione was coadministered, it was observed that the percentage loss of SNc somas and terminals was decreasing with increasing glutathione concentration (Figure 7D).
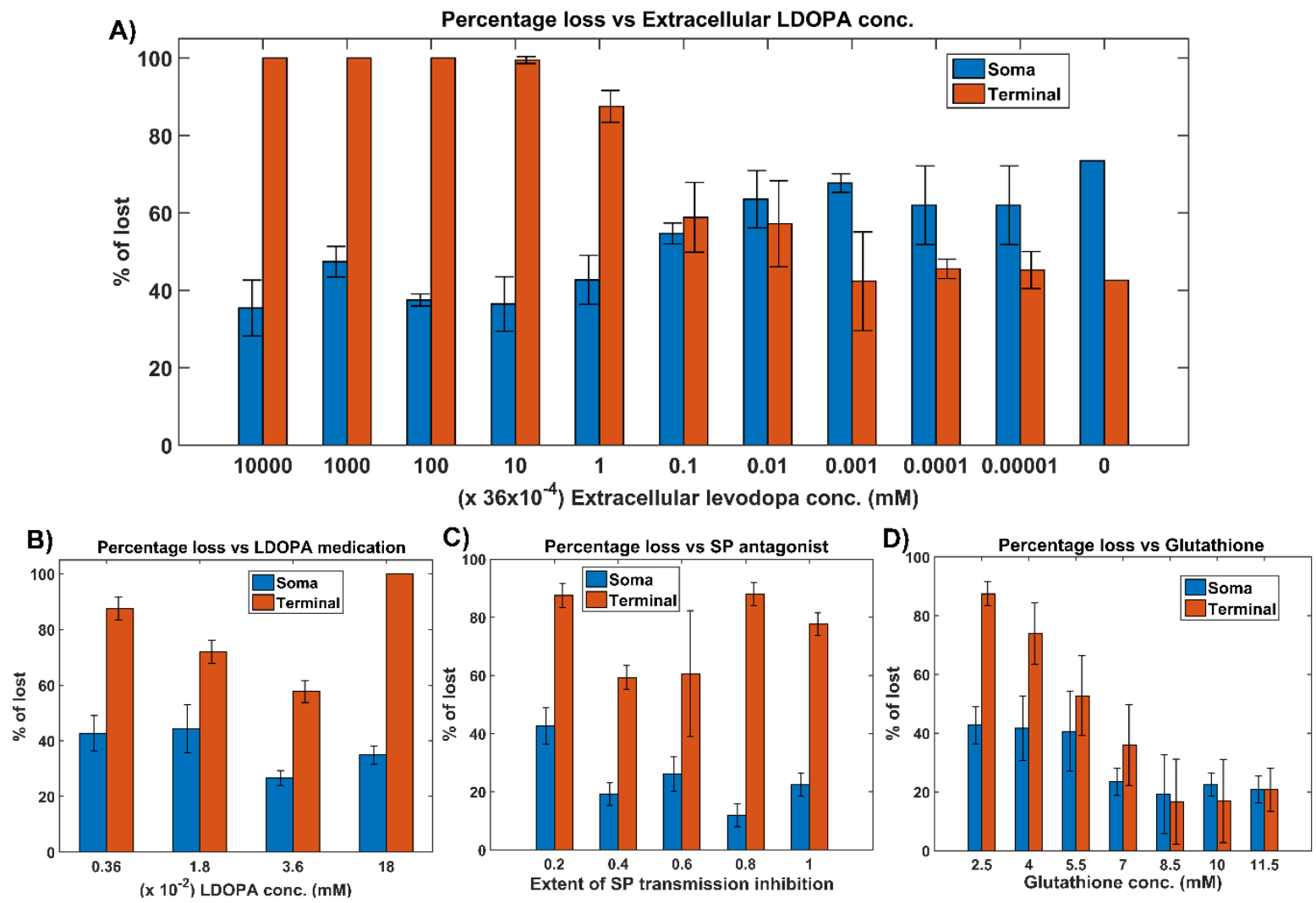

Figure 7: Model response to different therapeutics under energy deficiency. (A) Percentage loss for various extracellular LDOPA concentrations, (B) Percentage loss during LDOPA therapy, (C) Percentage loss during SP 
antagonist therapy, (D) Percentage loss during glutathione therapy. All the therapeutic interventions were initiated at 25\% soma or terminal loss. conc, concentration; LDOPA, levodopa; SP, substance P; mM, millimolar.

\section{DISCUSSION}

\subsection{LIT model}

The goal of this computational study is to develop a model of SNc-striatum, which helps us in understanding LDOPA-induced toxicity in SNc neurons under energy deficiency conditions. From both homogeneous and heterogeneous energy deficiency results, it suggests that SNc (axonal) terminals are more vulnerable to energy imbalance when compared to SNc cell bodies (somas) which was observed experimentally, where injury is initiated at axonal terminals (Burré et al., 2010; Cheng et al., 2010; Giguère et al., 2019; Wong et al., 2019). The higher positive currents from STN might lead to excitotoxic loss of SNc somas (Figure 5A, blue bar), and increased ROS production might lead to increased SNc terminal loss (Figure 5A, orange bar). DA transporters, which play a crucial role in DA neurotransmission, were depleted more in axonal terminals compared to cell bodies in early PD (Fazio et al., 2018). From these studies, it can be postulated that pathogenesis starts at axonal terminals, which are more vulnerable to energy deficiencies and therefore are ideal sites for developing novel disease-modifying therapeutics.

When lower concentrations of extracellular LDOPA were available, the loss of SNc somas was more when compared to SNc terminals (Figure 7A). This might be due to lower extracellular DA levels as a result of lower extracellular LDOPA concentrations and lower vesicular DA levels (due to reduced packing of DA into vesicles as a result of lower energy levels) causing disinhibition of SNc somas (as result of lesser cortical excitation of MSNs) which are already in a low energy state. Due to disinhibition and energy deficiency, SNc somas might become overactive, which leads to calcium build-up, resulting in excitotoxic loss of SNc somas (Albin and Greenamyre, 1992; Muddapu et al., 2019). When higher concentrations of extracellular LDOPA were available, the loss of SNc terminals was more when compared to SNc somas (Figure 7A). This might be due to higher cytoplasmic DA levels as a result of higher extracellular LDOPA concentrations, lower vesicular packing of DA (due to lower energy levels) and LDOPA-induced stimulation of DA metabolism (Mosharov et al., 2009) resulting in DA-mediated oxidative stress in the SNc terminals (Farooqui, 2012; Morrison et al., 2012). Due to higher DA levels and energy deficiency, DA in SNc terminals causes oxidative stress, which leads to SNc terminal loss. At higher concentrations of extracellular LDOPA, loss of SNc somas was lower compared to lower concentrations of extracellular LDOPA as a result of 
the restoration of inhibitory tone from MSNs due to higher extracellular DA concentrations. The extracellular LDOPA concentration ranging from $36 \times 10^{-7} \mathrm{mM}$ to $36 \times 10^{-4} \mathrm{mM}$ was considered as basal extracellular LDOPA concentrations in the proposed LIT model. At these values, the percentage loss of SNc somas and terminals was similar, which was observed in previous studies (Cullen and Wong-Lin, 2015; Khor and Hsu, 2007; Reed et al., 2012). Our model was able to show the significance of basal extracellular LDOPA concentrations, which needed to be maintained for normal functioning.

When external LDOPA concentration administered was in the range from $36 \times 10^{-4} \mathrm{mM}$ to $36 \times 10^{-3} \mathrm{mM}$, it was observed that the percentage loss of SNc somas and terminals was decreasing, which suggests the therapeutic benefits of LDOPA therapy in altering or halting the progression of the SNc cell loss. When external LDOPA concentration administered rated was above this range, the neuroprotective effect of LDOPA therapy diminished. From LDOPA+SP antagonist co-administration, it was observed that inhibiting excitotoxicity in SNc somas did not decrease SNc terminal loss, which suggests that excitotoxicity in $\mathrm{SNc}$ somas does not contribute to oxidative stress in $\mathrm{SNc}$ terminals in LDOPA-induced toxicity. From LDOPA+glutathione co-administration, it was observed that inhibiting oxidative stress in SNc terminals did decrease the loss of SNc somas, which suggests that oxidative stress in $\mathrm{SNc}$ terminals does contribute to excitotoxicity in $\mathrm{SNc}$ somas in LDOPA-induced toxicity. From these results, we can suggest that adjunct therapies such as antioxidants (Betharia et al., 2019; Borah and Mohanakumar, 2010; Carvey et al., 1997; Deng et al., 2020; Nikolova et al., 2019; Pardo et al., 1993, 1995; Walkinshaw and Waters, 1995) and other potential therapies such as D2 agonists (Asanuma et al., 2003), Glycogen synthase kinase 3 inhibitors (Choi and Koh, 2018), calcium-binding protein drugs (Isaacs et al., 1997), etc. co-administrated along with LDOPA should be able to evade LDOPA toxicity in all stages of PD.

From the simulation results, it was observed that the LDOPA-induced toxicity in cell bodies and axonal terminals of SNc neurons was autoxidation-irrelevant and autoxidationrelevant, respectively. In the case of cell bodies, excess DA in striatum due to LDOPA therapy stimulates glutamatergic cortical inputs to MSNs, which leads to overexcitation of MSNs. The overexcited MSNs co-release SP along with GABA onto SNc neurons. SP modulates SNc glutamatergic inputs in such a way that it overexcites SNc neurons resulting in excitotoxic neuronal loss in SNc. However, in the case of axonal terminals, excess DA in terminals due to LDOPA therapy leads to autooxidation of DA. The autoxidation of DA results in the production 
of free radicals, which lead to oxidative stress in SNc axonal terminals resulting in axonal synaptic pruning of SNc neurons. The study suggests that LDOPA-induced toxicity occurs by two mechanisms: DA-mediated oxidative stress in axonal terminals of SNc neurons and by exacerbating STN-mediated overexcitation in cell bodies of SNc neurons.

\subsection{SNc positive feedback loops - Scope of vulnerability}

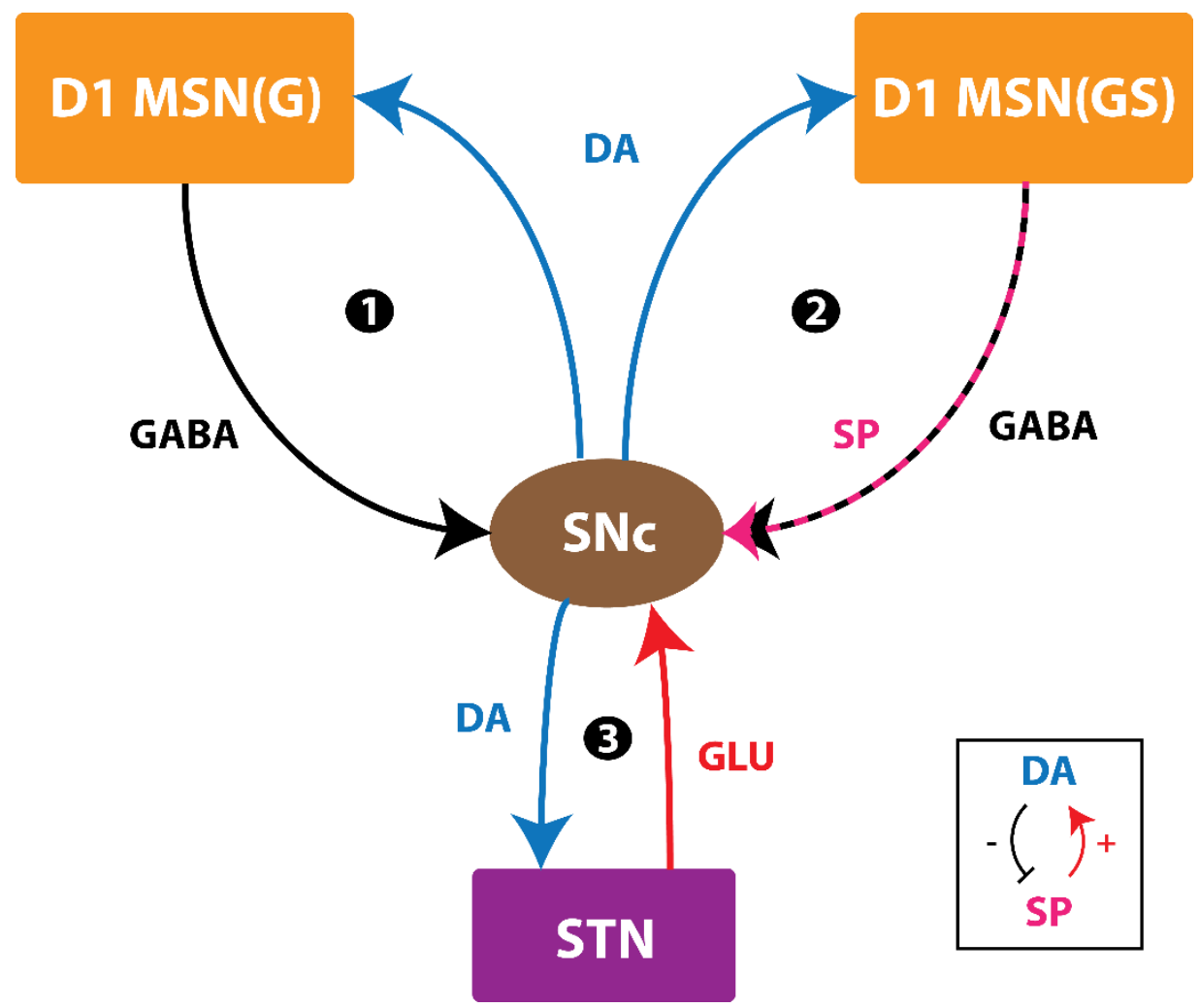

Figure 8: Positive feedback loops of SNc. SNc, substantia nigra pars compacta; STN, subthalamic nucleus; D1 $M S N(G)$, D1-type DA receptor-expressing medium spiny neuron (release GABA); D1 MSN(GS), D1-type DA receptor-expressing medium spiny neuron (co-release GABA and SP); DA, dopamine; SP, substance P; GLU, glutamate; GABA, gamma-aminobutyric acid. Inset, DA-SP feedback.

In normal conditions, there is no $\mathrm{SNc}$ cell or terminal loss where $\mathrm{SNc}$ maintains the dopaminergic tone on its target regions such as STN, D1-MSN(G), and D1-MSN(GS). In the first loop (Figure 8), normal dopaminergic tone to D1-MSN(G) results in inhibition of SNc by GABAergic projections. In the second loop (Figure 8), normal dopaminergic tone to D1MSN(GS) results in inhibition of SNc by GABA and lesser excitation of SNc by SP due to DA-SP feedback (Brimblecombe and Cragg, 2015; Thornton and Vink, 2015). In the third loop (Figure 8), normal dopaminergic tone to STN results in lesser excitation of SNc by glutamatergic projections (Hassani et al., 1997; Magill et al., 2001; Yang et al., 2016).

Under pathological conditions, there is SNc cell or terminal loss where SNc fails to maintain the dopaminergic tone in its target regions such as STN, D1-MSN(G), and D1- 
MSN(GS). In the first loop (Figure 8), DA deficiency in the striatum causes lesser excitation of D1-MSN(G) by cortex, which, by feedback, results in disinhibition of SNc. In other words, initial DA deficiency due to SNc cell loss causes lesser excitation of D1-MSN(G), which in turn disinhibits SNc resulting in further SNc cell loss due to excitotoxicity, which acts as positive feedback. In the second loop, DA deficiency in the striatum causes lesser excitation of D1-MSN(GS) by cortex, which results in disinhibition (through GABA) and further excitation (through SP; due to low DA, the effect of SP gets enhanced) of SNc. Thus, the disinhibition of $\mathrm{SNc}$ happens in a manner similar to the first loop; however, the overexcitation of SNc happens due to the DA-SP feedback mechanism, which also acts as positive feedback. In the third loop, DA deficiency causes overexcitation of STN, which results in overactivation of SNc. In other words, initial DA deficiency due to SNc cell loss causes overexcitation of STN, which in turn overexcites SNc, by a positive feedback mechanism, resulting in further SNc cell loss due to excitotoxicity.

In medication conditions, LDOPA is administrated where dopaminergic tone to SNc target regions (STN, D1-MSN(G), D1-MSN(GS)) is restored. If the administrated LDOPA dosage goes beyond a certain threshold, overexcited D1-MSN(GS) through the DA-SP feedback mechanism makes SNc hyperactive, which in turn results in SNc cell loss due to excitotoxicity. Along with SNc cell body loss, SNc terminals also undergo degeneration due to excess DA causing oxidative stress. To summarize, LDOPA-induced toxicity in SNc doesn't occur when LDOPA dosage is below the threshold, which results in the survival of remaining SNc cells. However, if, LDOPA dosage goes beyond a threshold, from that point onwards, the aforementioned runaway effect kicks in, leading to a progressive and irrevocable cell loss in SNc. Thus, it is evident that LDOPA might be toxic to SNc neurons under high dosage, which triggers a runaway effect resulting in uncontrollable SNc cell loss.

\subsection{Limitations and Future Directions}

Though the proposed model captures the exciting results of LDOPA-induced toxicity, it is not without limitations. In the proposed model, for example, the serotonergic system was not considered, which takes up LDOPA and contributes to striatal DA levels (Stansley and Yamamoto, 2015; Svenningsson et al., 2015) that contribute to LDOPA-induced dyskinesias (Carta et al., 2008; Carta and Tronci, 2014). Similarly, interneurons in the striatum were also not considered for simplifying the model. 
In the proposed model, the ischemic condition was implemented by lowering glucose and oxygen levels, which can be extended by adding a blood vessel module (Cloutier et al., 2009) and varying cerebral blood flow to simulate ischemia condition more realistically. In the proposed model, stress was monitored in SNc neurons alone, which can be extended to other neuronal types in the model by monitoring stress levels, where intracellular calcium build-up can be a stress indicator (Bano and Ankarcrona, 2018). In order to do so, all neuronal types should be modelled as conductance-based models where calcium dynamics should be included. From our studies, it is shown that LDOPA dosage plays an important role in the progression of the disease. The proposed model will be integrated with a behavioural model of cortico-basal ganglia circuitry (Muralidharan et al., 2018) to show the effect of LDOPA-induced toxicity at the behavioural level and optimize the LDOPA dosage so as to achieve maximum effect with a minimal dosage of the drug.

We suggest some experimental approaches to validate some of the predictions from our modelling study. Under induced progressive energy deficiency conditions in animal models (Puginier et al., 2019), LDOPA administration at moderate levels can also be toxic, which needs to be studied by measuring metabolites of DA autoxidation process. In order to study the effects of LDOPA-induced toxicity in SNc somas in midbrain and $\mathrm{SNc}$ terminals in the striatum, similar toxin-induced animal models can be used, where oxidative stress in terminals can be examined by monitoring the levels of free radicals and excitotoxicity in somas can be examined by monitoring calcium levels (Wong et al., 2019). By co-administering antioxidants along with LDOPA in toxin-induced animal models (Betharia et al., 2019; Borah and Mohanakumar, 2010; Carvey et al., 1997; Nikolova et al., 2019; Pardo et al., 1993, 1995; Walkinshaw and Waters, 1995), the progression of SNc soma and terminal loss can be altered along with prolonging the 'honeymoon period' of LDOPA therapy (Erro et al., 2016; Holford and Nutt, 2008; Stocchi et al., 2010).

\section{CONCLUSIONS}

In conclusion, we believe that the proposed model provides significant insights in understanding the mechanisms behind LDOPA-induced toxicity under energy deficiency conditions. From simulation results, it was shown that SNc terminals are more vulnerable to energy imbalances when compared to SNc somas. The study suggests that LDOPA-induced toxicity occurs differently in SNc somas and terminals: in the case of SNc somas, it might be due to excitotoxicity caused by STN, and in case of SNc terminals, it might be due to oxidative 
stress caused by dopamine autoxidation. From adjunct therapies, it was clear that coadministering antioxidants, along with LDOPA, can be neuroprotective. By the aforementioned modelling efforts in addition to some earlier ones (Muddapu et al., 2019), we are trying to understand the root cause of PD neurodegeneration as energy deficiency occurring at different neural hierarchies: subcellular, cellular and network levels.

\section{AUTHOR CONTRIBUTIONS}

VRM - Conceptualization; Model development; Data curation; Formal analysis; Investigation; Methodology; Manuscript writing; VSC - Conceptualization; Model development; Data curation; Formal analysis; Investigation; Methodology; Manuscript writing; Supervision; KV - Conceptualization; Model development; KR - Conceptualization; Model development;

\section{REFERENCES}

Agid, Y. (1998). Levodopa: Is toxicity a myth? Neurology 50, 858-863. doi:10.1212/WNL.50.4.858.

Albin, R. L., and Greenamyre, J. T. (1992). Alternative excitotoxic hypotheses. Neurology 42, 733-738. doi:10.1212/WNL.42.4.733.

Allers, K. A., Walters, J. R., and Kreiss, D. S. (2003). "Neuronal Firing Patterns in the Subthalamic Nucleus," in The Basal Ganglia V, eds. A. M. Graybiel, M. R. Delong, and S. T. Kitai (Boston, MA: Springer US), 245-254. doi:10.1007/978-1-4615-0179-4_25.

Arbuthnott, G. W., and Wickens, J. (2007). Space, time and dopamine. Trends Neurosci. 30, 62-69. doi:10.1016/j.tins.2006.12.003.

Ares-Santos, S., Granado, N., Espadas, I., Martinez-Murillo, R., and Moratalla, R. (2014). Methamphetamine Causes Degeneration of Dopamine Cell Bodies and Terminals of the Nigrostriatal Pathway Evidenced by Silver Staining. Neuropsychopharmacology 39, 1066-1080. doi:10.1038/npp.2013.307.

Asanuma, M., Miyazaki, I., and Ogawa, N. (2003). Dopamine- or L-DOPA-induced neurotoxicity: The role of dopamine quinone formation and tyrosinase in a model of Parkinson's disease. Neurotox. Res. 5, 165-176. doi:10.1007/BF03033137.

Bano, D., and Ankarcrona, M. (2018). Beyond the critical point: An overview of excitotoxicity, calcium overload and the downstream consequences. Neurosci. Lett. 663, 79-85. doi:10.1016/j.neulet.2017.08.048. 
Ben-Jonathan, N., and Hnasko, R. (2001). Dopamine as a prolactin (PRL) inhibitor. Endocr. Rev. 22, 724-763. doi:10.1210/edrv.22.6.0451.

Betharia, S., Rondón-Ortiz, A. N., and Brown, D. A. (2019). Disubstituted Dithiolethione ACDT Exerts Neuroprotective Effects Against 6-Hydroxydopamine-Induced Oxidative Stress in SH-SY5Y Cells. Neurochem. Res. 44, 1878-1892. doi:10.1007/s11064-01902823-3.

Beurrier, C., Congar, P., Bioulac, B., and Hammond, C. (1999). Subthalamic nucleus neurons switch from single-spike activity to burst-firing mode. J. Neurosci. 19, 599-609.

Billings, J. L., Gordon, S. L., Rawling, T., Doble, P. A., Bush, A. I., Adlard, P. A., et al. (2019). 1-3,4-dihydroxyphenylalanine (1-DOPA) modulates brain iron, dopaminergic neurodegeneration and motor dysfunction in iron overload and mutant alpha-synuclein mouse models of Parkinson's disease. J. Neurochem. 150, 88-106. doi:10.1111/jnc. 14676 .

Blomeley, C., and Bracci, E. (2008). Substance P depolarizes striatal projection neurons and facilitates their glutamatergic inputs. J. Physiol. 586, 2143-2155. doi:10.1113/jphysiol.2007.148965.

Blomeley, C. P., Kehoe, L. A., and Bracci, E. (2009). Substance P Mediates Excitatory Interactions between Striatal Projection Neurons. J. Neurosci. 29, 4953-4963. doi:10.1523/JNEUROSCI.6020-08.2009.

Bolam, J. P., and Pissadaki, E. K. (2012). Living on the edge with too many mouths to feed: Why dopamine neurons die. Mov. Disord. 27, 1478-1483. doi:10.1002/mds.25135.

Borah, A., and Mohanakumar, K. P. (2010). Salicylic acid protects against chronic 1-DOPAinduced 6-OHDA generation in experimental model of parkinsonism. Brain Res. 1344, 192-199. doi:10.1016/j.brainres.2010.05.010.

Brimblecombe, K. R., and Cragg, S. J. (2015). Substance P weights striatal dopamine transmission differently within the striosome-matrix axis. J. Neurosci. 35, 9017-9023. doi:10.1523/JNEUROSCI.0870-15.2015.

Bugaysen, J., Bronfeld, M., Tischler, H., Bar-Gad, I., and Korngreen, A. (2010). Electrophysiological Characteristics of Globus Pallidus Neurons. PLoS One 5, e12001. doi:10.1371/journal.pone.0012001. 
Burré, J., Sharma, M., Tsetsenis, T., Buchman, V., Etherton, M. R., and Südhof, T. C. (2010). $\alpha$-Synuclein promotes SNARE-complex assembly in vivo and in vitro. Science (80-. ). 329, 1663-1667. doi:10.1126/science.1195227.

Buxton, D., Bracci, E., Overton, P. G., and Gurney, K. (2017). Striatal Neuropeptides Enhance Selection and Rejection of Sequential Actions. Front. Comput. Neurosci. 11, 62. doi:10.3389/fncom.2017.00062.

Cadet, J. L., Jayanthi, S., and Deng, X. (2003). Speed kills: Cellular and molecular bases of methamphetamine-induced nerve terminal degeneration and neuronal apoptosis. FASEB J. 17, 1775-1788. doi:10.1096/fj.03-0073rev.

Camargo, S. M. R., Vuille-dit-Bille, R. N., Mariotta, L., Ramadan, T., Huggel, K., Singer, D., et al. (2014). The Molecular Mechanism of Intestinal Levodopa Absorption and Its Possible Implications for the Treatment of Parkinson's Disease. J. Pharmacol. Exp. Ther. 351, 114-123. doi:10.1124/jpet.114.216317.

Carta, M., Carlsson, T., Muñoz, A., Kirik, D., and Björklund, A. (2008). Serotonin-dopamine interaction in the induction and maintenance of L-DOPA-induced dyskinesias. Prog. Brain Res. 172, 465-78. doi:10.1016/S0079-6123(08)00922-9.

Carta, M., and Tronci, E. (2014). Serotonin System Implication in 1-DOPA-Induced Dyskinesia: From Animal Models to Clinical Investigations. Front. Neurol. 5, 78. doi:10.3389/fneur.2014.00078.

Carvey, P. M., Pieri, S., and Ling, Z. D. (1997). Attenuation of levodopa-induced toxicity in mesencephalic cultures by pramipexole. J. Neural Transm. 104, 209-228. doi:10.1007/BF01273182.

Caudle, W. M., Richardson, J. R., Wang, M. Z., Taylor, T. N., Guillot, T. S., McCormack, A. L., et al. (2007). Reduced vesicular storage of dopamine causes progressive nigrostriatal neurodegeneration. J. Neurosci. 27, 8138-8148. doi:10.1523/JNEUROSCI.031907.2007.

Cepeda, C., Buchwald, N. A., and Levine, M. S. (1993). Neuromodulatory actions of dopamine in the neostriatum are dependent upon the excitatory amino acid receptor subtypes activated. Proc. Natl. Acad. Sci. 90, 9576-9580. doi:10.1073/pnas.90.20.9576.

Chen, K. C., and Budygin, E. A. (2007). Extracting the basal extracellular dopamine 
concentrations from the evoked responses: Re-analysis of the dopamine kinetics. $J$. Neurosci. Methods 164, 27-42. doi:10.1016/j.jneumeth.2007.03.020.

Cheng, H. C., Ulane, C. M., and Burke, R. E. (2010). Clinical progression in Parkinson disease and the neurobiology of axons. Ann. Neurol. 67, 715-725. doi:10.1002/ana.21995.

Cheng, N. N., Maeda, T., Kume, T., Kaneko, S., Kochiyama, H., Akaike, A., et al. (1996). Differential neurotoxicity induced by L-DOPA and dopamine in cultured striatal neurons. Brain Res. 743, 278-283. doi:10.1016/S0006-8993(96)01056-6.

Choi, H., and Koh, S.-H. (2018). Understanding the role of glycogen synthase kinase-3 in LDOPA-induced dyskinesia in Parkinson's disease. Expert Opin. Drug Metab. Toxicol. 14, 83-90. doi:10.1080/17425255.2018.1417387.

Chou, T.-C. (1976). Derivation and properties of Michaelis-Menten type and Hill type equations for reference ligands. J. Theor. Biol. 59, 253-276. doi:10.1016/00225193(76)90169-7.

Cloutier, M., Bolger, F. B., Lowry, J. P., and Wellstead, P. (2009). An integrative dynamic model of brain energy metabolism using in vivo neurochemical measurements. $J$. Comput. Neurosci. 27, 391-414. doi:10.1007/s10827-009-0152-8.

Cullen, M., and Wong-Lin, K. (2015). Integrated dopaminergic neuronal model with reduced intracellular processes and inhibitory autoreceptors. IET Syst. Biol. 9, 245-258. doi:10.1049/iet-syb.2015.0018.

De Vito, M. J., and Wagner, G. C. (1989). Methamphetamine-induced neuronal damage: A possible role for free radicals. Neuropharmacology 28, 1145-1150. doi:10.1016/00283908(89)90130-5.

Dedman, J. R., and Kaetzel, M. A. (1997). Chapter 22 The role of intracellular calcium as a regulatory signal. Princ. Med. Biol. 7, 515-530. doi:10.1016/S1569-2582(97)80130-4.

Degenetais, E. (2002). Electrophysiological Properties of Pyramidal Neurons in the Rat Prefrontal Cortex: An In Vivo Intracellular Recording Study. Cereb. Cortex 12, 1-16. doi:10.1093/cercor/12.1.1.

Deng, H., Jia, Y., Pan, D., and Ma, Z. (2020). Berberine alleviates rotenone-induced cytotoxicity by antioxidation and activation of PI3K/Akt signaling pathway in SH-SY5Y 
cells. Neuroreport 31, 41-47. doi:10.1097/WNR.0000000000001365.

Elias, S., Ritov, Y., and Bergman, H. (2008). Balance of Increases and Decreases in Firing Rate of the Spontaneous Activity of Basal Ganglia High-Frequency Discharge Neurons. J. Neurophysiol. 100, 3086-3104. doi:10.1152/jn.90714.2008.

Erro, R., Picillo, M., Vitale, C., Amboni, M., Moccia, M., Santangelo, G., et al. (2016). The non-motor side of the honeymoon period of Parkinson's disease and its relationship with quality of life: a 4-year longitudinal study. Eur. J. Neurol. 23, 1673-1679. doi:10.1111/ene.13106.

Fahn, S. (1997). Levodopa-induced neurotoxicity: Does it represent a problem for the treatment of Parkinson's disease? CNS Drugs 8, 376-393. doi:10.2165/00023210199708050-00004.

Fahn, S. (2005). Does levodopa slow or hasten the rate of progression of Parkinson's disease? J. Neurol. 252, iv37-iv42. doi:10.1007/s00415-005-4008-5.

Fahn, S., Oakes, D., Shoulson, I., Kieburtz, K., Rudolph, A., Lang, A., et al. (2004). Levodopa and the Progression of Parkinson's Disease. N. Engl. J. Med. 351, 2498-2508. doi:10.1056/NEJMoa033447.

Farooqui, T. (2012). "Dopamine-Mediated Oxidative Stress Associated with Neurodegeneration in Parkinson Disease," in Molecular Aspects of Neurodegeneration and Neuroprotection, ed. T. Farooqui (BENTHAM SCIENCE PUBLISHERS), 62-71. doi:10.2174/978160805092511101010062.

Fazio, P., Svenningsson, P., Cselényi, Z., Halldin, C., Farde, L., and Varrone, A. (2018). Nigrostriatal dopamine transporter availability in early Parkinson's disease. Mov. Disord. 33, 592-599. doi:10.1002/mds.27316.

Figura, M., Kuśmierska, K., Bucior, E., Szlufik, S., Koziorowski, D., Jamrozik, Z., et al. (2018). Serum amino acid profile in patients with Parkinson's disease. PLoS One 13, e0191670. doi:10.1371/journal.pone.0191670.

Ford, C. P. (2014). The role of D2-autoreceptors in regulating dopamine neuron activity and transmission. Neuroscience 282, 13-22. doi:10.1016/j.neuroscience.2014.01.025.

Fu, H., Hardy, J., and Duff, K. E. (2018). Selective vulnerability in neurodegenerative diseases. Nat. Neurosci. 21, 1350-1358. doi:10.1038/s41593-018-0221-2. 
Garris, P. A., Christensen, J. R., Rebec, G. V., and Wightman, R. M. (1997). Real-time measurement of electrically evoked extracellular dopamine in the striatum of freely moving rats. J. Neurochem. 68, 152-61. doi:10.1046/j.1471-4159.1997.68010152.x.

Gerfen, C. R. (1985). The neostriatal mosaic. I. compartmental organization of projections from the striatum to the substantia nigra in the rat. J. Comp. Neurol. 236, 454-476. doi:10.1002/cne.902360404.

Giguère, N., Delignat-Lavaud, B., Herborg, F., Voisin, A., Li, Y., Jacquemet, V., et al. (2019). Increased vulnerability of nigral dopamine neurons after expansion of their axonal arborization size through D2 dopamine receptor conditional knockout. PLOS Genet. 15, e1008352. doi:10.1371/journal.pgen.1008352.

Goldman, J. G., and Postuma, R. (2014). Premotor and nonmotor features of Parkinson's disease. Curr. Opin. Neurol. 27, 434-441. doi:10.1097/WCO.0000000000000112.

Gonon, F. G. (1988). Nonlinear relationship between impulse flow and dopamine released by rat midbrain dopaminergic neurons as studied by in vivo electrochemistry. Neuroscience 24, 19-28. Available at: http://www.ncbi.nlm.nih.gov/pubmed/3368048.

Götz, T., Kraushaar, U., Geiger, J., Lübke, J., Berger, T., and Jonas, P. (1997). Functional properties of AMPA and NMDA receptors expressed in identified types of basal ganglia neurons. J. Neurosci. 17, 204-15. Available at: http://www.ncbi.nlm.nih.gov/pubmed/8987749.

Grace, A. A., and Bunney, B. S. (1984a). The control of firing pattern in nigral dopamine neurons: burst firing. J. Neurosci. 4, 2877-2890. doi:6150071.

Grace, A. A., and Bunney, B. S. (1984b). The control of firing pattern in nigral dopamine neurons: single spike firing. J. Neurosci. 4, 2866-76. doi:6150070.

Griffith, J. S., and Horn, G. (1966). An analysis of spontaneous impulse activity of units in the striate cortex of unrestrained cats. J. Physiol. 186, 516-534. doi:10.1113/jphysiol.1966.sp008053.

Guo, J., Zhao, X., Li, Y., Li, G., and Liu, X. (2018). Damage to dopaminergic neurons by oxidative stress in Parkinson's disease (Review). Int. J. Mol. Med. 41, 1817-1825. doi:10.3892/ijmm.2018.3406.

Hassani, O. K., François, C., Yelnik, J., and Féger, J. (1997). Evidence for a dopaminergic 
innervation of the subthalamic nucleus in the rat. Brain Res. 749, 88-94. Available at: http://www.ncbi.nlm.nih.gov/pubmed/9070631.

Hauser, R. A., Lyons, K. E., McClain, T., Carter, S., and Perlmutter, D. (2009). Randomized, double-blind, pilot evaluation of intravenous glutathione in Parkinson's disease. Mov. Disord. 24, 979-983. doi:10.1002/mds.22401.

Hebb, M. O., and Robertson, H. A. (1999). Identification of a subpopulation of substantia nigra pars compacta ??- aminobutyric acid neurons that is regulated by basal ganglia activity. J. Comp. Neurol. 416, 30-44. doi:10.1002/(SICI)10969861(20000103)416:1<30::AID-CNE4>3.0.CO;2-2.

Hegeman, D. J., Hong, E. S., Hernández, V. M., and Chan, C. S. (2016). The external globus pallidus: Progress and perspectives. Eur. J. Neurosci. 43, 1239-1265. doi:10.1111/ejn.13196.

Holford, N., and Nutt, J. G. (2008). Disease progression, drug action and Parkinson's disease: why time cannot be ignored. Eur. J. Clin. Pharmacol. 64, 207-16. doi:10.1007/s00228007-0427-9.

Humphries, M. D., Lepora, N., Wood, R., and Gurney, K. (2009). Capturing dopaminergic modulation and bimodal membrane behaviour of striatal medium spiny neurons in accurate, reduced models. Front. Comput. Neurosci. 3, 26. doi:10.3389/neuro.10.026.2009.

Isaacs, K. R., Wolpoe, M. E., and Jacobowitz, D. M. (1997). Calretinin-immunoreactive dopaminergic neurons from embryonic rat mesencephalon are resistant to levodopainduced neurotoxicity. Exp. Neurol. 146, 25-32. doi:10.1006/exnr.1997.6530.

Izhikevich, E. M. (2003). Simple model of spiking neurons. IEEE Trans. Neural Networks 14, 1569-1572. doi:10.1109/TNN.2003.820440.

Jankovic, J., and Aguilar, L. G. (2008). Current approaches to the treatment of Parkinson's disease. Neuropsychiatr. Dis. Treat. 4, 743-57. doi:10.2147/ndt.s2006.

Jenner, P. G., and Brin, M. F. (1998). Levodopa neurotoxicity: experimental studies versus clinical relevance. Neurology 50, S39-43; discussion S44-8. doi:10.1212/wnl.50.6_suppl_6.s39.

Johnson, M. B., Young, A. D., and Marriott, I. (2017). The therapeutic potential of targeting 
substance P/NK-1R interactions in inflammatory CNS disorders. Front. Cell. Neurosci. 10, 1-14. doi:10.3389/fncel.2016.00296.

Kariya, S., Takahashi, N., Hirano, M., and Ueno, S. (2005). Increased vulnerability to LDOPA toxicity in dopaminergic neurons from VMAT2 heterozygote knockout mice. $J$. Mol. Neurosci. 27, 277-279. doi:10.1385/JMN:27:3:277.

Khor, S.-P., and Hsu, A. (2007). The Pharmacokinetics and Pharmacodynamics of Levodopa in the Treatment of Parkinsons Disease. Curr. Clin. Pharmacol. 2, 234-243. doi:10.2174/157488407781668802.

Kita, H., and Kita, T. (2011). Role of Striatum in the Pause and Burst Generation in the Globus Pallidus of 6-OHDA-Treated Rats. Front. Syst. Neurosci. 5, 42. doi:10.3389/fnsys.2011.00042.

Koch, K. W., and Fuster, J. M. (1989). Unit activity in monkey parietal cortex related to haptic perception and temporary memory. Exp. Brain Res. 76, 292-306. doi:10.1007/BF00247889.

Kuznetsova, A. Y., Huertas, M. A., Kuznetsov, A. S., Paladini, C. A., and Canavier, C. C. (2010). Regulation of firing frequency in a computational model of a midbrain dopaminergic neuron. J. Comput. Neurosci. 28, 389-403. doi:10.1007/s10827-0100222-y.

Larsen, K. E., Fon, E. A., Hastings, T. G., Edwards, R. H., and Sulzer, D. (2002). Methamphetamine-induced degeneration of dopaminergic neurons involves autophagy and upregulation of dopamine synthesis. J. Neurosci. 22, 8951-8960.

Lessard, A., and Pickel, V. M. (2005). Subcellular distribution and plasticity of neurokinin-1 receptors in the rat substantia nigra and ventral tegmental area. Neuroscience 135, 13091323. doi:10.1016/j.neuroscience.2005.07.025.

Li, X.-Y., Mei, G.-H., Dong, Q., Zhang, Y., Guo, Z.-L., Su, J.-J., et al. (2015). Enhanced Neuroprotective Effects of Coadministration of Tetrandrine with Glutathione in Preclinical Model of Parkinson's Disease. Parkinsons. Dis. 2015, 931058. doi:10.1155/2015/931058.

Lindahl, M., Kamali Sarvestani, I., Ekeberg, O., and Kotaleski, J. H. (2013). Signal enhancement in the output stage of the basal ganglia by synaptic short-term plasticity in 
the direct, indirect, and hyperdirect pathways. Front. Comput. Neurosci. 7, 76. doi:10.3389/fncom.2013.00076.

Lipski, J., Nistico, R., Berretta, N., Guatteo, E., Bernardi, G., and Mercuri, N. B. (2011). LDOPA: a scapegoat for accelerated neurodegeneration in Parkinson's disease? Prog. Neurobiol. 94, 389-407. doi:10.1016/j.pneurobio.2011.06.005.

Lotharius, J., Falsig, J., Van Beek, J., Payne, S., Dringen, R., Brundin, P., et al. (2005). Progressive degeneration of human mesencephalic neuron-derived cells triggered by dopamine-dependent oxidative stress is dependent on the mixed-lineage kinase pathway. J. Neurosci. 25, 6329-6342. doi:10.1523/JNEUROSCI.1746-05.2005.

Luo, Y., and Roth, G. S. (2000). The Roles of Dopamine Oxidative Stress and Dopamine Receptor Signaling in Aging and Age-Related Neurodegeneration. Antioxid. Redox Signal. 2, 449-460. doi:10.1089/15230860050192224.

Magill, P. J., Bolam, J. P., and Bevan, M. D. (2001). Dopamine regulates the impact of the cerebral cortex on the subthalamic nucleus-globus pallidus network. Neuroscience 106, 313-330. doi:10.1016/S0306-4522(01)00281-0.

Mahon, S., Vautrelle, N., Pezard, L., Slaght, S. J., Deniau, J.-M., Chouvet, G., et al. (2006). Distinct Patterns of Striatal Medium Spiny Neuron Activity during the Natural SleepWake Cycle. J. Neurosci. 26, 12587-12595. doi:10.1523/JNEUROSCI.3987-06.2006.

Malhotra, J. D., and Kaufman, R. J. (2011). ER stress and its functional link to mitochondria: role in cell survival and death. Cold Spring Harb. Perspect. Biol. 3, a004424. doi:10.1101/cshperspect.a004424.

Mandali, A., Rengaswamy, M., Chakravarthy, V. S., and Moustafa, A. A. (2015). A spiking Basal Ganglia model of synchrony, exploration and decision making. Front. Neurosci. 9, 191. doi:10.3389/fnins.2015.00191.

Mantyh, P. W., Maggio, J. E., and Hunt, S. P. (1984). The autoradiographic distribution of kassinin and substance $\mathrm{K}$ binding sites is different from the distribution of substance $\mathrm{P}$ binding sites in rat brain. Eur. J. Pharmacol. 102, 361-4. doi:10.1016/00142999(84)90269-3.

Mark, K. A., Soghomonian, J. J., and Yamamoto, B. K. (2004). High-dose methamphetamine acutely-activates the striatonigral pathway to increase striatal glutamate and mediate 
long-term dopamine toxicity. J. Neurosci. 24, 11449-11456.

doi:10.1523/JNEUROSCI.3597-04.2004.

Masato, A., Plotegher, N., Boassa, D., and Bubacco, L. (2019). Impaired dopamine metabolism in Parkinson's disease pathogenesis. Mol. Neurodegener. 14, 1-21. doi:10.1186/s13024-019-0332-6.

Melamed, E., Offen, D., Shirvan, A., Djaldetti, R., Barzilai, A., and Ziv, I. (1998). Levodopa Toxicity and Apoptosis. Ann. Neurol. 44, S149-S154. doi:10.1002/ana.410440722.

Michmizos, K. P., and Nikita, K. S. (2011). Addition of deep brain stimulation signal to a local field potential driven Izhikevich model masks the pathological firing pattern of an STN neuron. in 2011 Annual International Conference of the IEEE Engineering in Medicine and Biology Society (Boston, MA: IEEE), 7290-7293. doi:10.1109/IEMBS.2011.6091700.

Mingazov, E. R., and Ugryumov, M. V. (2019). Molecular Markers of Dopamine Transport in Nigrostriatal Dopaminergic Neurons as an Index of Neurodegeneration and Neuroplasticity. Neurochem. J. 13, 43-48. doi:10.1134/S181971241901015X.

Mischley, L. K., Lau, R. C., Shankland, E. G., Wilbur, T. K., and Padowski, J. M. (2017). Phase IIb Study of Intranasal Glutathione in Parkinson's Disease. J. Parkinsons. Dis. 7, 289-299. doi:10.3233/JPD-161040.

Miyazaki, I., and Asanuma, M. (2008). Dopaminergic neuron-specific oxidative stress caused by dopamine itself. Acta Med. Okayama 62, 141-50. doi:10.18926/AMO/30942.

Morrison, B. E., Marcondes, M. C. G., Nomura, D. K., Sanchez-Alavez, M., SanchezGonzalez, A., Saar, I., et al. (2012). Cutting Edge: IL-13R $\alpha 1$ Expression in Dopaminergic Neurons Contributes to Their Oxidative Stress-Mediated Loss following Chronic Peripheral Treatment with Lipopolysaccharide. J. Immunol. 189, 5498-5502. doi:10.4049/jimmunol.1102150.

Mosharov, E. V., Larsen, K. E., Kanter, E., Phillips, K. A., Wilson, K., Schmitz, Y., et al. (2009). Interplay between Cytosolic Dopamine, Calcium, and $\alpha$-Synuclein Causes Selective Death of Substantia Nigra Neurons. Neuron 62, 218-229. doi:10.1016/J.NEURON.2009.01.033.

Muddapu, V. R., and Chakravarthy, V. S. (2020a). A Multi-Scale Computational Model of 
Excitotoxic Loss of Dopaminergic Cells in Parkinson's Disease. bioRxiv, 2020.02.20.957704. doi:10.1101/2020.02.20.957704.

Muddapu, V. R., and Chakravarthy, V. S. (2020b). Influence of Energy Deficiency on the Molecular Processes of Substantia Nigra Pars Compacta Cell for Understanding Parkinsonian Neurodegeneration - A Comprehensive Biophysical Computational Model. bioRxiv, 2020.02.18.950337. doi:10.1101/2020.02.18.950337.

Muddapu, V. R., Dharshini, S. A. P., Chakravarthy, V. S., and Gromiha, M. M. (2020). Neurodegenerative diseases -Is metabolic deficiency the root cause? Front. Neurosci. 14, 213. doi:10.3389/FNINS.2020.00213.

Muddapu, V. R., Mandali, A., Chakravarthy, V. S., and Ramaswamy, S. (2019). A Computational Model of Loss of Dopaminergic Cells in Parkinson's Disease Due to Glutamate-Induced Excitotoxicity. Front. Neural Circuits 13, 11. doi:10.3389/fncir.2019.00011.

Müller, T., Hefter, H., Hueber, R., Jost, W. H., Leenders, K. L., Odin, P., et al. (2004). Is levodopa toxic? J. Neurol. Suppl. 251, 44-46. doi:10.1007/s00415-004-1610-x.

Muralidharan, V., Mandali, A., Balasubramani, P. P., Mehta, H., Srinivasa Chakravarthy, V., and Jahanshahi, M. (2018). “A Cortico-Basal Ganglia Model to Understand the Neural Dynamics of Targeted Reaching in Normal and Parkinson's Conditions," in Computational Neuroscience Models of the Basal Ganglia, eds. V. S. Chakravarthy and A. A. Moustafa (Singapore: Springer Singapore), 167-195. doi:10.1007/978-981-108494-2_10.

Murer, M. G., Dziewczapolski, G., Menalled, L. B., García, M. C., Agid, Y., Gershanik, O., et al. (1998). Chronic levodopa is not toxic for remaining dopamine neurons, but instead promotes their recovery, in rats with moderate nigrostriatal lesions. Ann. Neurol. 43, 561-575. doi:10.1002/ana.410430504.

Murer, M. G., Raisman-Vozari, R., and Gershanik, O. (1999). Levodopa in Parkinson's disease: Neurotoxicity issue laid to rest? Drug Saf. 21, 339-352. doi:10.2165/00002018199921050-00001.

Nikolova, G., Karamalakova, Y., and Gadjeva, V. (2019). Reducing oxidative toxicity of Ldopa in combination with two different antioxidants: an essential oil isolated from Rosa 
Damascena Mill., and vitamin C. Toxicol. reports 6, 267-271. doi:10.1016/j.toxrep.2019.03.006.

Olanow, C. W., and Obeso, J. A. (2011). Levodopa toxicity and Parkinson disease: still a need for equipoise. Neurology 77, 1416-7. doi:10.1212/WNL.0b013e318232ac0a.

Oorschot, D. E. (1996). Total number of neurons in the neostriatal, pallidal, subthalamic, and substantia nigral nuclei of the rat basal ganglia: A stereological study using the cavalieri and optical disector methods. J. Comp. Neurol. 366, 580-599. doi:10.1002/(SICI)10969861(19960318)366:4<580::AID-CNE3>3.0.CO;2-0.

Orrenius, S., Zhivotovsky, B., and Nicotera, P. (2003). Regulation of cell death: the calciumapoptosis link. Nat. Rev. Mol. Cell Biol. 4, 552-65. doi:10.1038/nrm1150.

Pacelli, C., Giguère, N., Bourque, M. J., Lévesque, M., Slack, R. S., and Trudeau, L. É. (2015). Elevated Mitochondrial Bioenergetics and Axonal Arborization Size Are Key Contributors to the Vulnerability of Dopamine Neurons. Curr. Biol. 25, 2349-2360. doi:10.1016/j.cub.2015.07.050.

Paoletti, F. P., Tambasco, N., and Parnetti, L. (2019). Levodopa treatment in Parkinson's disease: earlier or later? Ann. Transl. Med. 7, S189-S189. doi:10.21037/atm.2019.07.36.

Pardo, B., Mena, M. A., Casarejos, M. J., Paíno, C. L., and De Yébenes, J. G. (1995). Toxic effects of L-DOPA on mesencephalic cell cultures: protection with antioxidants. Brain Res. 682, 133-143. doi:10.1016/0006-8993(95)00341-M.

Pardo, B., Mena, M. A., Fahn, S., and de Yébenes, J. G. (1993). Ascorbic acid protects against levodopa-induced neurotoxicity on a catecholamine-rich human neuroblastoma cell line. Mov. Disord. 8, 278-284. doi:10.1002/mds.870080305.

Pifl, C., Rajput, A., Reither, H., Blesa, J., Cavada, C., Obeso, J. A., et al. (2014). Is Parkinson's Disease a Vesicular Dopamine Storage Disorder? Evidence from a Study in Isolated Synaptic Vesicles of Human and Nonhuman Primate Striatum. J. Neurosci. 34, 8210-8218. doi:10.1523/JNEUROSCI.5456-13.2014.

Pissadaki, E. K., and Bolam, J. P. (2013). The energy cost of action potential propagation in dopamine neurons: clues to susceptibility in Parkinson's disease. Front. Comput.

Neurosci. 7, 13. doi:10.3389/fncom.2013.00013.

Pitcher, T. L., Wickens, J. R., and Reynolds, J. N. J. (2007). Differences in striatal spiny 
neuron action potentials between the spontaneously hypertensive and Wistar-Kyoto rat strains. Neuroscience 146, 135-142. doi:10.1016/j.neuroscience.2007.01.003.

Poewe, W., Antonini, A., Zijlmans, J. C., Burkhard, P. R., and Vingerhoets, F. (2010). Levodopa in the treatment of Parkinson's disease: an old drug still going strong. Clin. Interv. Aging 5, 229-38. doi:10.2147/cia.s6456.

Porenta, G., and Riederer, P. (1982). A Mathematical Model of the Dopaminergic Synapse: Stability and Sensitivity Analyses, and Simulation of Parkinson's Disease and Aging Processes. Cybern. Syst. 13, 257-274. doi:10.1080/01969728208927705.

Puginier, E., Bharatiya, R., Chagraoui, A., Manem, J., Cho, Y. H., Garret, M., et al. (2019). Early neurochemical modifications of monoaminergic systems in the R6/1 mouse model of Huntington's disease. Neurochem. Int. 128, 186-195. doi:10.1016/J.NEUINT.2019.05.001.

Reed, M. C., Nijhout, H. F., and Best, J. A. (2012). Mathematical Insights into the Effects of Levodopa. Front. Integr. Neurosci. 6, 1-24. doi:10.3389/fnint.2012.00021.

Reiner, A., Hart, N. M., Lei, W., and Deng, Y. (2010). Corticostriatal projection neurons Dichotomous types and dichotomous functions. Front. Neuroanat. 4, 1-15. doi:10.3389/fnana.2010.00142.

Ribeiro-da-Silva, A., and Hökfelt, T. (2000). Neuroanatomical localisation of substance P in the CNS and sensory neurons. Neuropeptides 34, 256-271. doi:10.1054/npep.2000.0834.

Ricaurte, G. A., Guillery, R. W., Seiden, L. S., Schuster, C. R., and Moore, R. Y. (1982). Dopamine nerve terminal degeneration produced by high doses of methylamphetamine in the rat brain. Brain Res. 235, 93-103. doi:10.1016/0006-8993(82)90198-6.

Ricaurte, G. A., Seiden, L. S., and Schuster, C. R. (1984). Further evidence that amphetamines produce long-lasting dopamine neurochemical deficits by destroying dopamine nerve fibers. Brain Res. 303, 359-364. doi:10.1016/0006-8993(84)91221-6.

Rubin, J. E., and Terman, D. (2004). High frequency stimulation of the subthalamic nucleus eliminates pathological thalamic rhythmicity in a computational model. J. Comput. Neurosci. 16, 211-235. doi:10.1023/B:JCNS.0000025686.47117.67.

Schapira, A. H. V. (2008). The clinical relevance of levodopa toxicity in the treatment of 
Parkinson's disease. Mov. Disord. 23. doi:10.1002/mds.22146.

Shimozawa, A., Fujita, Y., Kondo, H., Takimoto, Y., Terada, M., Sanagi, M., et al. (2019).

Effect of 1-dopa/benserazide on propagation of pathological $\alpha$-synuclein. Front.

Neurosci. 13, 1-8. doi:10.3389/fnins.2019.00595.

Sivam, S. P. (1991). Dopamine dependent decrease in enkephalin and substance P levels in basal ganglia regions of postmortem Parkinsonian brains. Neuropeptides 18, 201-207. doi:10.1016/0143-4179(91)90148-C.

Stansley, B., and Yamamoto, B. (2015). L-Dopa and Brain Serotonin System Dysfunction. Toxics 3, 75-88. doi:10.3390/toxics3010075.

Stocchi, F., Jenner, P., and Obeso, J. A. (2010). When Do Levodopa Motor Fluctuations First Appear in Parkinson's Disease. Eur. Neurol. 63, 257-266. doi:10.1159/000300647.

Surmeier, D. J., Ding, J., Day, M., Wang, Z., and Shen, W. (2007). D1 and D2 dopaminereceptor modulation of striatal glutamatergic signaling in striatal medium spiny neurons. Trends Neurosci. 30, 228-235. doi:10.1016/j.tins.2007.03.008.

Surmeier, D. J., Guzman, J. N., Sanchez-Padilla, J., and Goldberg, J. A. (2011). The origins of oxidant stress in parkinson's disease and therapeutic strategies. Antioxidants Redox Signal. 14, 1289-1301. doi:10.1089/ars.2010.3521.

Sutoo, D., Yabe, K., and Akiyama, K. (1999). Quantitative imaging of substance P in the human brain using a brain mapping analyzer. Neurosci. Res. 35, 339-346. doi:10.1016/S0168-0102(99)00101-7.

Svenningsson, P., Rosenblad, C., Arvidsson, K. A. E., Wictorin, K., Keywood, C., Shankar, B., et al. (2015). Eltoprazine counteracts 1-DOPA-induced dyskinesias in Parkinson's disease: A dose-finding study. Brain 138, 963-973. doi:10.1093/brain/awu409.

Takashima, H., Tsujihata, M., Kishikawa, M., and Freed, W. J. (1999). Bromocriptine protects dopaminergic neurons from levodopa-induced toxicity by stimulating D2receptors. Exp. Neurol. 159, 98-104. doi:10.1006/exnr.1999.7122.

Tepper, J. M., and Lee, C. R. (2007). GABAergic control of substantia nigra dopaminergic neurons. Prog. Brain Res. 160, 189-208. doi:10.1016/S0079-6123(06)60011-3.

Thanvi, B. R., and Lo, T. C. N. (2004). Long term motor complications of levodopa: clinical 
features, mechanisms, and management strategies. Postgrad. Med. J. 80, 452-8. doi:10.1136/pgmj.2003.013912.

Thornton, E., Tran, T. T. B., and Vink, R. (2010). A substance P mediated pathway contributes to 6-hydroxydopamine induced cell death. Neurosci. Lett. 481, 64-67. doi:10.1016/j.neulet.2010.06.057.

Thornton, E., and Vink, R. (2012). Treatment with a Substance P Receptor Antagonist Is Neuroprotective in the Intrastriatal 6-Hydroxydopamine Model of Early Parkinson's Disease. PLoS One 7, e34138. doi:10.1371/journal.pone.0034138.

Thornton, E., and Vink, R. (2015). Substance P and its tachykinin NK1 receptor: a novel neuroprotective target for Parkinson's disease. Neural Regen. Res. 10, 1403-5. doi:10.4103/1673-5374.165505.

Tripathy, S. J., Burton, S. D., Geramita, M., Gerkin, R. C., and Urban, N. N. (2015). Brainwide analysis of electrophysiological diversity yields novel categorization of mammalian neuron types. J. Neurophysiol. 113, 3474-3489. doi:10.1152/jn.00237.2015.

Tripathy, S. J., Savitskaya, J., Burton, S. D., Urban, N. N., and Gerkin, R. C. (2014). NeuroElectro: a window to the world's neuron electrophysiology data. Front. Neuroinform. 8, 40. doi:10.3389/fninf.2014.00040.

Vandecasteele, M., Glowinski, J., and Venance, L. (2005). Electrical Synapses between dopaminergic neurons of the substantia nigra pars compacta. J Neurosci 25, 291-298. doi:Doi 10.1523/Jneurosci.4167-04.2005.

Walkinshaw, G., and Waters, C. M. (1995). Induction of apoptosis in catecholaminergic PC12 cells by L-DOPA. Implications for the treatment of Parkinson's disease. J. Clin. Invest. 95, 2458-2464. doi:10.1172/JCI117946.

Wang, Q., Chu, C.-H., Qian, L., Chen, S.-H., Wilson, B., Oyarzabal, E., et al. (2014). Substance P Exacerbates Dopaminergic Neurodegeneration through Neurokinin-1 Receptor-Independent Activation of Microglial NADPH Oxidase. J. Neurosci. 34, 12490-12503. doi:10.1523/JNEUROSCI.2238-14.2014.

Weiner, W. J. (2006). Levodopa--toxic or neuroprotective? Nat. Clin. Pract. Neurol. 2, 5189. doi:10.1038/ncpneuro0293.

Wojda, U., Salinska, E., and Kuznicki, J. (2008). Calcium ions in neuronal degeneration. 
IUBMB Life 60, 575-590. doi:10.1002/iub.91.

Wong, Y. C., Luk, K., Purtell, K., Burke Nanni, S., Stoessl, A. J., Trudeau, L., et al. (2019). Neuronal vulnerability in Parkinson disease: Should the focus be on axons and synaptic terminals? Mov. Disord. 34, 1406-1422. doi:10.1002/mds.27823.

Yang, C., Yan, Z., Zhao, B., Wang, J., Gao, G., Zhu, J., et al. (2016). D2 dopamine receptors modulate neuronal resonance in subthalamic nucleus and cortical high-voltage spindles through HCN channels. Neuropharmacology 105, 258-269. doi:10.1016/j.neuropharm.2016.01.026.

Zeevalk, G. D., Razmpour, R., and Bernard, L. P. (2008). Glutathione and Parkinson's disease: Is this the elephant in the room? Biomed. Pharmacother. 62, 236-249. doi:10.1016/j.biopha.2008.01.017.

Ziv, I., Zilkha-Falb, R., Offen, D., Shirvan, A., Barzilai, A., and Melamed, E. (1997). Levodopa induces apoptosis in cultured neuronal cells-A possible accelerator of nigrostriatal degeneration in Parkinson's disease? Mov. Disord. 12, 17-23. doi:10.1002/mds.870120105. 
Table 1: Different population sizes in the proposed LIT model (Oorschot, 1996).

\begin{tabular}{|c|c|}
\hline Network type & Size (\# of nodes) \\
\hline SNc (soma) & $8 \times 8(64)$ \\
\hline SNc (terminal) & $32 \times 32(1024)$ \\
\hline D1-MSN (G) & $32 \times 32(1024)$ \\
\hline D1-MSN (GS) & $32 \times 32(1024)$ \\
\hline STN & $32 \times 32(1024)$ \\
\hline GPe & $32 \times 32(1024)$ \\
\hline CTX & $32 \times 32(1024)$ \\
\hline
\end{tabular}

\section{Reference:}

Oorschot, D. E. (1996). Total number of neurons in the neostriatal, pallidal, subthalamic, and substantia nigral nuclei of the rat basal ganglia: A stereological study using the cavalieri and optical disector methods. J. Comp. Neurol. 366, 580-599. doi:10.1002/(SICI)10969861(19960318)366:4<580::AID-CNE3>3.0.CO;2-0. 
Table 1: Parameter values used in the proposed model of LIT (Humphries et al., 2009;

Izhikevich, 2003; Muddapu et al., 2019).

\begin{tabular}{|c|c|c|c|c|}
\hline Parameter(s) & STN & GPe & CTX & MSN \\
\hline \multicolumn{5}{|l|}{ Izhikevich parameters } \\
\hline$a\left(m s^{-1}\right)$, & $a=0.005$ & $a=0.1$ & $a=0.03$ & $a=0.01$ \\
\hline$b\left(p A \cdot m V^{-1}\right)$ & $b=0.265$ & $b=0.2$ & $b=-2$ & $b=-20$ \\
\hline$c(m V)$ & $c=-65$ & $c=-65$ & $c=-50$ & $c=-55$ \\
\hline$d(p A)$ & $d=1.5$ & $d=2$ & $d=100$ & $d=91$ \\
\hline External current $\left(I^{x}\right)$ & $3 p A$ & $4.25 p A$ & $100 p A$ & $0 p A$ \\
\hline Maximum peak of voltage $\left(v_{\text {peak }}^{x}\right)$ & $30 \mathrm{mV}$ & $30 \mathrm{mV}$ & $40 \mathrm{mV}$ & $35 \mathrm{mV}$ \\
\hline Membrane capacitance $\left(C^{x}\right)$ & $1 \mu F$ & $1 \mu F$ & $100 \mu F$ & $15.2 p F$ \\
\hline Resting potential $\left(v_{r}^{x}\right)$ & - & - & - & $-80 m V$ \\
\hline Threshold potential $\left(v_{t}^{x}\right)$ & - & - & - & $-29.7 m V$ \\
\hline Membrane constant $\left(k^{x}\right)$ & - & - & - & $1 p A \cdot m V^{-1}$ \\
\hline Number of laterals $\left(\right.$ nlat $\left.^{x}\right)$ & 11 & 15 & - & - \\
\hline Radius of Gaussian laterals $\left(R^{x}\right)$ & 1.4 & 1.6 & - & - \\
\hline Synaptic strength within laterals $\left(A^{x}\right)$ & 1.3 & 0.1 & - & - \\
\hline Time decay constant for AMPA $\left(\tau_{A M P A}\right)$ & $6 \mathrm{~ms}$ & $6 m s$ & $6 \mathrm{~ms}$ & $6 \mathrm{~ms}$ \\
\hline Time decay constant for NMDA $\left(\tau_{N M D A}\right)$ & $160 \mathrm{~ms}$ & $160 \mathrm{~ms}$ & $160 \mathrm{~ms}$ & $160 \mathrm{~ms}$ \\
\hline Time decay constant for GABA $\left(\tau_{G A B A}\right)$ & $4 m s$ & $4 m s$ & $4 m s$ & $4 \mathrm{~ms}$ \\
\hline $\begin{array}{l}\text { Synaptic potential of AMPA receptor } \\
\left(E_{A M P A}\right)\end{array}$ & $0 \mathrm{mV}$ & $0 \mathrm{mV}$ & $0 \mathrm{mV}$ & $0 \mathrm{mV}$ \\
\hline $\begin{array}{l}\text { Synaptic potential of NMDA receptor } \\
\left(E_{N M D A}\right)\end{array}$ & $0 \mathrm{mV}$ & $0 \mathrm{mV}$ & $0 \mathrm{mV}$ & $0 \mathrm{mV}$ \\
\hline $\begin{array}{l}\text { Synaptic potential of GABA receptor } \\
\left(E_{G A B A}\right)\end{array}$ & $-60 m V$ & $-60 m V$ & $-60 m V$ & $-60 m V$ \\
\hline
\end{tabular}




$1 \mathrm{mM}$

$1 m M$

\begin{tabular}{l|l}
$1 m M$ & $1 m M$
\end{tabular}

\section{References:}

Humphries, M. D., Lepora, N., Wood, R., and Gurney, K. (2009). Capturing dopaminergic modulation and bimodal membrane behaviour of striatal medium spiny neurons in accurate, reduced models. Front. Comput. Neurosci. 3, 26. doi:10.3389/neuro.10.026.2009.

Izhikevich, E. M. (2003). Simple model of spiking neurons. IEEE Trans. Neural Networks 14, 1569-1572. doi:10.1109/TNN.2003.820440.

Muddapu, V. R., Mandali, A., Chakravarthy, V. S., and Ramaswamy, S. (2019). A Computational Model of Loss of Dopaminergic Cells in Parkinson's Disease Due to Glutamate-Induced Excitotoxicity. Front. Neural Circuits 13, 11. doi:10.3389/fncir.2019.00011. 
Table 1: Connectivity patterns in the proposed LIT model (Oorschot, 1996).

\begin{tabular}{|c|c|}
\hline From - to & Pattern (signal) \\
\hline $\mathrm{SNc}$ (soma) - SNc (terminal) & 1 to 16 (Calcium) \\
\hline SNc (terminal) - D1-MSN (GS) & 20 to 1 (Dopamine) \\
\hline SNc (terminal) - D1-MSN (G) & 20 to 1 (Dopamine) \\
\hline D1-MSN (GS) - D1-MSN (G) & 1 to 1 (GABA \& SP) \\
\hline D1-MSN (G) - D1-MSN (GS) & 1 to 1 (GABA) \\
\hline D1-MSN (GS) - SNc (soma) & 200 to $1(\mathrm{GABA} \& \mathrm{SP})$ \\
\hline D1-MSN (G) - SNc (soma) & 200 to 1 (GABA) \\
\hline STN - GPe & 1 to 1 (Glutamate) \\
\hline $\mathrm{GPe}-\mathrm{STN}$ & 1 to 1 (GABA) \\
\hline $\mathrm{STN}-\mathrm{SNc}$ (soma) & 16 to 1 (Glutamate) \\
\hline CTX - D1-MSN (GS) & 1 to 1 (Glutamate) \\
\hline CTX - D1-MSN (G) & 1 to 1 (Glutamate) \\
\hline STN - STN & Gaussian neighborhoods (Glutamate) \\
\hline $\mathrm{GPe}-\mathrm{GPe}$ & Gaussian neighborhoods (GABA) \\
\hline $\mathrm{SNc}-\mathrm{SNc}$ & Gaussian neighborhoods (GABA) \\
\hline
\end{tabular}

\section{Reference:}

Oorschot, D. E. (1996). Total number of neurons in the neostriatal, pallidal, subthalamic, and substantia nigral nuclei of the rat basal ganglia: A stereological study using the cavalieri and optical disector methods. J. Comp. Neurol. 366, 580-599. doi:10.1002/(SICI)10969861(19960318)366:4<580::AID-CNE3>3.0.CO;2-0. 
Table 1: Parameter values used in the proposed model of LIT (Buxton et al., 2017; Reed et al., 2012; Terman et al., 2002).

\begin{tabular}{|c|c|c|c|}
\hline Parameter & Value & Parameter & Value \\
\hline Number of laterals $\left(\right.$ nlat $\left.^{x}\right)$ & 5 & $\theta_{g}$ & $20 \mathrm{mV}$ \\
\hline $\begin{array}{l}\text { Radius of Gaussian laterals } \\
\left(R^{x}\right)\end{array}$ & 1.6 & $\theta_{g}^{H}$ & $-57 m V$ \\
\hline $\begin{array}{l}\text { Synaptic strength within } \\
\text { laterals }\left(A^{x}\right)\end{array}$ & 0.1 & $\sigma_{g}^{H}$ & $2 m V$ \\
\hline Synaptic conductance $\left(W_{x \rightarrow y}\right)$ & 0.01 & $\alpha$ & $2 m s^{-1}$ \\
\hline $\begin{array}{l}\text { Synaptic potential of GABA } \\
\text { receptor }\left(E_{G A B A}\right)\end{array}$ & $63.45 \mathrm{mV}$ & $\beta$ & $0.08 m s^{-1}$ \\
\hline$s_{\max }^{S T N}$ & 1.3 & $c d_{s t n}$ & 4.87 \\
\hline$s_{\text {min }}^{G P e}$ & 0.1 & $c d_{\text {gpe }}$ & 7 \\
\hline$S_{\min }^{S N C}$ & $1 \times 10^{-6}$ & $c d_{s n c}$ & 4.6055 \\
\hline$c d 2$ & 0.1 & $w_{s p}$ & 5000 \\
\hline$K^{M S N}$ & 0.0289 & $L^{M S N}$ & 0.331 \\
\hline$\alpha_{D A}^{D 1-M S N(G)}$ & 1 & $\alpha_{D A}^{D 1-M S N(G S)}$ & 2 \\
\hline$w_{G P e \rightarrow G P e}$ & 1 & $w_{S N C \rightarrow S N C}$ & 0.01 \\
\hline$w_{S T N \rightarrow G P e}$ & 1 & $w_{G P e \rightarrow S T N}$ & 20 \\
\hline$w_{S T N \rightarrow S T N}$ & 1.3 & $w_{S T N \rightarrow S N C}$ & 0.3 \\
\hline$W_{D 1-M S N}(G) \rightarrow S N C$ & 0.5 & $W_{D 1-M S N}(G S) \rightarrow S N C$ & 0.5 \\
\hline$W_{D 1-M S N}(G) \rightarrow D 1-M S N(G S)$ & 500 & $w_{C T X \rightarrow D 1-M S N}(G S)$ & 100 \\
\hline$w_{C T X \rightarrow D 1-M S N}(G)$ & 100 & $\tau_{d}^{s p}$ & $40 \mathrm{~ms}$ \\
\hline
\end{tabular}




\begin{tabular}{|c|c|c|c|}
\hline$\tau_{f}^{s p}$ & $200 \mathrm{~ms}$ & $\tau_{r}^{s p}$ & $10 \mathrm{~ms}$ \\
\hline$\beta_{s p}$ & 0.47 & $\lambda_{s p}$ & 5.5 \\
\hline$b_{s p}$ & 2.5 & $F_{S T N \rightarrow S N C}$ & $1 \times 10^{-5}$ \\
\hline$F_{D 1-M S N}(G) \rightarrow S N C$ & $4.15 \times 10^{-6}$ & $F_{D 1-M S N}(G S) \rightarrow S N C$ & $4.15 \times 10^{-6}$ \\
\hline$V_{\text {trans }}^{\max }$ & $5.11 \times 10^{-7} \mathrm{mM} \cdot \mathrm{ms}^{-1}$ & $K_{m}^{L D O P A_{S}}$ & $0.032 \mathrm{mM}$ \\
\hline$\left[T Y R_{S}\right]$ & $0.063 \mathrm{mM}$ & $K_{a}^{T Y R_{S}}$ & $0.064 \mathrm{mM}$ \\
\hline$\left[T R P_{S}\right]$ & $0.082 \mathrm{mM}$ & $K_{a}^{T R P_{S}}$ & $0.015 \mathrm{mM}$ \\
\hline$P_{\text {soma }}^{\text {snc }}$ & 64 & $P_{\text {terminal }}^{\text {Snc }}$ & 1024 \\
\hline$E R_{\text {thres }}$ & $2.15 \times 10^{-3} \mathrm{mM}$ & $R_{\text {thres }}$ & $0.0147 \mathrm{mM}$ \\
\hline$M T_{\text {thres }}$ & $0.0215 \mathrm{mM}$ & & \\
\hline
\end{tabular}

\section{References:}

Buxton, D., Bracci, E., Overton, P. G., and Gurney, K. (2017). Striatal Neuropeptides Enhance Selection and Rejection of Sequential Actions. Front. Comput. Neurosci. 11, 62. doi:10.3389/fncom.2017.00062.

Reed, M. C., Nijhout, H. F., and Best, J. A. (2012). Mathematical Insights into the Effects of Levodopa. Front. Integr. Neurosci. 6, 1-24. doi:10.3389/fnint.2012.00021.

Terman, D., Rubin, J. E., Yew, a C., and Wilson, C. J. (2002). Activity patterns in a model for the subthalamopallidal network of the basal ganglia. J. Neurosci. 22, 2963-76. doi:20026266. 